\title{
Estimation of Galactic Model Parameters in High Latitudes with SDSS
}

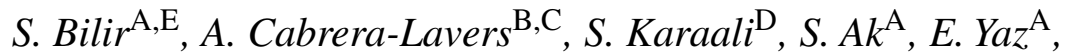 \\ and M. López-Corredoira ${ }^{\mathrm{B}}$ \\ A Istanbul University Science Faculty, Department of Astronomy and Space Sciences, \\ 34119, University-Istanbul, Turkey \\ B Instituto de Astrofísica de Canarias, E-38205 La Laguna, Tenerife, Spain \\ ${ }^{\mathrm{C}}$ GTC Project Office, E-38205 La Laguna, Tenerife, Spain \\ D Beykent University, Faculty of Science and Letters, Department of Mathematics \\ and Computing, Ayazağa 34396, Istanbul, Turkey \\ ${ }^{\mathrm{E}}$ Corresponding author. Email: sbilir@istanbul.edu.tr
}

Received 2007 August 3, accepted 2008 April 14

\begin{abstract}
We estimated the Galactic model parameters for a set of 36 high-latitude fields included in the currently available Data Release 5 (DR 5) of the Sloan Digital Sky Survey (SDSS), to explore their possible variation with the Galactic longitude. The thick disc scaleheight moves from $\sim 550 \mathrm{pc}$ at $120<l<150^{\circ}$ to $\sim 720 \mathrm{pc}$ at $250<l<290^{\circ}$, while the thin disc scaleheight is as large as $\sim 195 \mathrm{pc}$ in the anticenter direction and $\sim 15 \%$ lower at $|l|<30^{\circ}$. Finally, the axis ratio $(c / a)$ of the halo changes from a mean value of $\sim 0.55$ in the two first quadrants of the Galaxy to $\sim 0.70$ at $190<l<300^{\circ}$. For the halo, the reason for the dependence of the model parameters on the Galactic longitude arises from the well known asymmetric structure of this component. However, the variation of the model parameters of the thin and thick discs with Galactic longitude originates from the gravitational effect of the Galactic long bar. Moreover, the excess of stars in quadrant I (quadrant III) over quadrant IV (quadrant II) is in agreement with this scenario.
\end{abstract}

Keywords: Galaxy: disc — Galaxy: structure — Galaxy: fundamental parameters

\section{Introduction}

Traditional star-count analyses of the Galactic structure have provided a picture of the basic structural and stellar populations of the Galaxy. Examples and reviews of these analyses can be found in Bahcall (1986), Gilmore, Wyse \& Kuijken (1989), Majewski (1993), Robin, Reylé \& Crézé (2000) and recently Chen et al. (2001) and Siegel et al. (2002). The largest of the observational studies prior to the SDSS (York et al. 2000) are based on photographic surveys. The Basle Halo Program has presented the largest systematic photometric survey of the Galaxy (Becker 1965; Fenkart 1989a,b,c,d). The Basle Halo Program photometry is currently being re-calibrated and re-analysed, using an improved calibration of the $R G U$ photometric system (Buser \& Fenkart 1990; Buser, Rong \& Karaali 1998, 1999). More recent and future studies are being based on charge-coupled device (CCD) survey data.

Our knowledge of the structure of the Galaxy, as inferred from star count data with colour information, entered now to the next level of precision with the advent of new surveys such as SDSS, 2MASS, CADIS, BATC, DENIS, UKIDSS/VISTA, CFH/Megacam, and Suprime. Researchers have used different methods to determine the Galactic model parameters. In Table 1 of Karaali, Bilir \& Hamzaoğlu (2004) we can find an exhaustive list of the different values obtained for the structural parameters of the discs and halo of the Milky Way. One can see directly that there is a refinement in the numerical values of the model parameters. The local space density and the scaleheight of the thick disc can be given as an example. The evaluation of the thick disc have steadily moved towards shorter scaleheights, from 1.45 to $0.65 \mathrm{kpc}$ (Gilmore \& Reid 1983; Chen et al. 2001), and higher local densities (2-10\%). In many studies the range of values for the parameters is large. For example, Chen et al. (2001) and Siegel et al. (2002) give 6.5-13 and 6-10\%, respectively, for the local space density for the thick disc. However, one expects the most accurate numerical values for these recent works. That is, either the range for the parameters should be small or a single value with a small error should be given for each of them. It seems that workers have not been able to choose the most appropriate procedures in this topic.

Large range or different numerical values for a specific Galactic model parameter as estimated by different researchers may be due to several reasons:

1. The Galactic model parameters are Galactic latitude/ longitude dependent. The two works of Buser et al. (1998, 1999) confirm this suggestion. Although these authors give a mean value for each parameter, there are differences between the values of a given parameter for different fields. Also, it has been recently shown that 
Table 1. Galactic model parameters for 36 SDSS high-latitude fields

\begin{tabular}{|c|c|c|c|c|c|c|c|c|}
\hline \multirow{2}{*}{$\begin{array}{l}\langle l\rangle \\
(\mathrm{deg})\end{array}$} & \multirow{2}{*}{$\begin{array}{c}\text { Thin disc } \\
h_{\mathrm{z}, 1} \\
(\mathrm{pc})\end{array}$} & \multicolumn{2}{|c|}{ Thick disc } & \multicolumn{2}{|c|}{ Halo } & \multirow{2}{*}{$\begin{array}{c}N \\
\left(\operatorname{stars~deg}^{-2}\right)\end{array}$} & \multirow[t]{2}{*}{$\tilde{\chi}_{\min }^{2}$} & \multirow[t]{2}{*}{ Probability } \\
\hline & & $\begin{array}{l}h_{\mathrm{z}, 2} \\
(\mathrm{pc})\end{array}$ & $\begin{array}{c}n_{2} / n_{1} \\
(\%)\end{array}$ & $\begin{array}{c}n_{3} / n_{1} \\
(\%)\end{array}$ & $c / a$ & & & \\
\hline 0 & $177 \pm 9$ & $634 \pm 46$ & $10.96 \pm 1.23$ & $0.14 \pm 0.01$ & $0.60 \pm 0.02$ & $3672 \pm 61$ & 0.517 & 0.972 \\
\hline 10 & $178 \pm 6$ & $613 \pm 28$ & $12.50 \pm 0.90$ & $0.16 \pm 0.01$ & $0.58 \pm 0.01$ & $3686 \pm 61$ & 0.488 & 0.981 \\
\hline 20 & $167 \pm 8$ & $586 \pm 27$ & $15.14 \pm 1.15$ & $0.17 \pm 0.01$ & $0.57 \pm 0.01$ & $3527 \pm 59$ & 0.438 & 0.991 \\
\hline 30 & $187 \pm 9$ & $620 \pm 44$ & $11.67 \pm 1.33$ & $0.17 \pm 0.01$ & $0.56 \pm 0.01$ & $3391 \pm 58$ & 0.844 & 0.677 \\
\hline 40 & $180 \pm 6$ & $595 \pm 28$ & $13.46 \pm 1.46$ & $0.19 \pm 0.02$ & $0.54 \pm 0.02$ & $3370 \pm 58$ & 0.475 & 0.984 \\
\hline 50 & $172 \pm 6$ & $609 \pm 26$ & $13.30 \pm 0.89$ & $0.16 \pm 0.01$ & $0.58 \pm 0.02$ & $3251 \pm 57$ & 0.537 & 0.965 \\
\hline 60 & $195 \pm 6$ & $641 \pm 35$ & $10.33 \pm 0.86$ & $0.13 \pm 0.01$ & $0.62 \pm 0.01$ & $3008 \pm 55$ & 0.795 & 0.742 \\
\hline 70 & $181 \pm 7$ & $616 \pm 38$ & $11.07 \pm 1.01$ & $0.12 \pm 0.01$ & $0.62 \pm 0.01$ & $2733 \pm 52$ & 0.335 & 0.999 \\
\hline 80 & $178 \pm 7$ & $598 \pm 35$ & $10.86 \pm 1.03$ & $0.14 \pm 0.01$ & $0.58 \pm 0.01$ & $2514 \pm 50$ & 0.399 & 0.995 \\
\hline 90 & $192 \pm 7$ & $647 \pm 52$ & $7.89 \pm 1.02$ & $0.12 \pm 0.01$ & $0.60 \pm 0.02$ & $2252 \pm 47$ & 0.541 & 0.963 \\
\hline 100 & $189 \pm 5$ & $638 \pm 35$ & $7.94 \pm 0.69$ & $0.12 \pm 0.01$ & $0.58 \pm 0.01$ & $2063 \pm 45$ & 0.310 & 0.999 \\
\hline 110 & $186 \pm 4$ & $648 \pm 35$ & $7.11 \pm 0.62$ & $0.12 \pm 0.01$ & $0.59 \pm 0.01$ & $1932 \pm 44$ & 0.479 & 0.983 \\
\hline 120 & $196 \pm 7$ & $667 \pm 68$ & $5.45 \pm 0.90$ & $0.15 \pm 0.01$ & $0.54 \pm 0.01$ & $1807 \pm 43$ & 0.855 & 0.662 \\
\hline 130 & $184 \pm 6$ & $581 \pm 36$ & $8.75 \pm 0.96$ & $0.16 \pm 0.01$ & $0.54 \pm 0.01$ & $1732 \pm 42$ & 0.468 & 0.986 \\
\hline 140 & $172 \pm 4$ & $550 \pm 23$ & $10.26 \pm 0.70$ & $0.16 \pm 0.01$ & $0.54 \pm 0.01$ & $1698 \pm 41$ & 0.413 & 0.994 \\
\hline 150 & $183 \pm 4$ & $568 \pm 26$ & $8.71 \pm 0.69$ & $0.16 \pm 0.01$ & $0.54 \pm 0.01$ & $1712 \pm 41$ & 0.450 & 0.989 \\
\hline 160 & $195 \pm 8$ & $603 \pm 64$ & $7.23 \pm 1.22$ & $0.16 \pm 0.03$ & $0.53 \pm 0.04$ & $1697 \pm 41$ & 0.543 & 0.962 \\
\hline 170 & $195 \pm 7$ & $638 \pm 67$ & $6.35 \pm 1.05$ & $0.17 \pm 0.01$ & $0.54 \pm 0.01$ & $1719 \pm 41$ & 0.816 & 0.715 \\
\hline 180 & $195 \pm 4$ & $658 \pm 42$ & $6.22 \pm 0.62$ & $0.14 \pm 0.01$ & $0.61 \pm 0.03$ & $1766 \pm 42$ & 0.681 & 0.869 \\
\hline 190 & $198 \pm 4$ & $646 \pm 37$ & $6.46 \pm 0.59$ & $0.12 \pm 0.01$ & $0.65 \pm 0.01$ & $1822 \pm 43$ & 0.630 & 0.912 \\
\hline 200 & $199 \pm 7$ & $657 \pm 61$ & $6.35 \pm 0.91$ & $0.13 \pm 0.01$ & $0.67 \pm 0.03$ & $1911 \pm 44$ & 0.541 & 0.963 \\
\hline 210 & $179 \pm 5$ & $598 \pm 31$ & $9.46 \pm 0.77$ & $0.14 \pm 0.01$ & $0.71 \pm 0.02$ & $2083 \pm 46$ & 0.396 & 0.996 \\
\hline 220 & $185 \pm 7$ & $594 \pm 46$ & $9.16 \pm 1.12$ & $0.13 \pm 0.01$ & $0.76 \pm 0.02$ & $2186 \pm 47$ & 0.656 & 0.892 \\
\hline 230 & $198 \pm 4$ & $654 \pm 36$ & $7.08 \pm 0.59$ & $0.13 \pm 0.01$ & $0.75 \pm 0.01$ & $2314 \pm 48$ & 0.372 & 0.997 \\
\hline 240 & $174 \pm 4$ & $603 \pm 26$ & $9.18 \pm 0.57$ & $0.13 \pm 0.01$ & $0.71 \pm 0.04$ & $2264 \pm 48$ & 0.495 & 0.979 \\
\hline 250 & $189 \pm 4$ & $621 \pm 27$ & $8.09 \pm 0.56$ & $0.16 \pm 0.01$ & $0.64 \pm 0.01$ & $2310 \pm 48$ & 0.331 & 0.999 \\
\hline 260 & $188 \pm 6$ & $658 \pm 44$ & $7.53 \pm 0.79$ & $0.12 \pm 0.01$ & $0.72 \pm 0.02$ & $2439 \pm 49$ & 0.538 & 0.964 \\
\hline 270 & $186 \pm 9$ & $684 \pm 80$ & $6.82 \pm 1.23$ & $0.11 \pm 0.01$ & $0.73 \pm 0.03$ & $2545 \pm 50$ & 0.399 & 0.995 \\
\hline 280 & $196 \pm 4$ & $716 \pm 35$ & $6.00 \pm 0.46$ & $0.11 \pm 0.01$ & $0.73 \pm 0.02$ & $2728 \pm 52$ & 0.392 & 0.996 \\
\hline 290 & $188 \pm 9$ & $704 \pm 71$ & $6.85 \pm 1.09$ & $0.10 \pm 0.01$ & $0.75 \pm 0.02$ & $2967 \pm 54$ & 0.501 & 0.977 \\
\hline 300 & $190 \pm 7$ & $679 \pm 50$ & $7.60 \pm 0.89$ & $0.11 \pm 0.01$ & $0.73 \pm 0.01$ & $3133 \pm 56$ & 0.495 & 0.979 \\
\hline 310 & $173 \pm 3$ & $649 \pm 16$ & $9.33 \pm 0.42$ & $0.12 \pm 0.01$ & $0.68 \pm 0.01$ & $3258 \pm 57$ & 0.390 & 0.996 \\
\hline 320 & $186 \pm 8$ & $669 \pm 43$ & $8.32 \pm 0.86$ & $0.12 \pm 0.01$ & $0.68 \pm 0.01$ & $3465 \pm 59$ & 0.444 & 0.990 \\
\hline 330 & $178 \pm 7$ & $600 \pm 34$ & $10.74 \pm 1.04$ & $0.07 \pm 0.01$ & $0.66 \pm 0.01$ & $3590 \pm 60$ & 0.564 & 0.953 \\
\hline 340 & $184 \pm 5$ & $619 \pm 14$ & $10.30 \pm 0.82$ & $0.15 \pm 0.01$ & $0.61 \pm 0.01$ & $3554 \pm 60$ & 0.398 & 0.995 \\
\hline 350 & $200 \pm 6$ & $658 \pm 39$ & $8.73 \pm 0.89$ & $0.17 \pm 0.01$ & $0.58 \pm 0.01$ & $3689 \pm 61$ & 0.732 & 0.817 \\
\hline 360 & $177 \pm 9$ & $634 \pm 46$ & $10.96 \pm 1.23$ & $0.14 \pm 0.01$ & $0.60 \pm 0.01$ & $3672 \pm 61$ & 0.517 & 0.972 \\
\hline
\end{tabular}

the Galactic model parameters are Galactic longitude dependent (Bilir et al. 2006a,c; Cabrera-Lavers et al. 2007; Ak et al. 2007a).

2. The Galactic model parameters are absolute magnitude dependent (Karaali et al. 2004; Bilir, Karaali \& Gilmore 2006b). Hence, any procedure which excludes this argument gives Galactic model parameters spread in a large range.

3. Galactic model parameters change with limiting distance of completeness. That is, a specific model parameter is not the same for each set of Galactic model parameters estimated for different volumes (Karaali et al. 2007).

The difference between the Galactic model parameters estimated for fields with different Galactic latitudes and longitudes can be explained by the influence of the disc flaring and warping. The disc of our Galaxy is far from being radially smooth and uniform. On the contrary, its overall shape presents strong asymmetries. While the warp bends the Galactic plane upwards in the first and second Galactic longitude quadrants $\left(0 \leq l \leq 180^{\circ}\right)$ and downwards in the third and fourth quadrants $\left(180 \leq l \leq 360^{\circ}\right)$, the flare changes the scaleheight as a function of radial distance.

This warp is present in all Galactic components: dust (Drimmel \& Spergel 2001; Marshall et al. 2006), gas (Burton 1988, p295; Drimmel \& Spergel 2001; Nakanishi \& Sofue 2003; Levine, Blitz \& Heiles 2006; Voskes \& Burton 2006) and stars (López-Corredoira et al. 2002; Momany et al. 2006). All these components have the same node position, and their distributions are asymmetric. However, the amplitude of the dust warp seems to be less pronounced than the stellar and gaseous warps, that share the same approximate amplitude (López-Corredoira et al. 2002; Momany et al. 2006).

The stellar and gaseous flarings for the Milky Way are also compatible (Momany et al. 2006), showing that 
$h_{\mathrm{z}}$ increases with the galactocentric radius for $R>5 \mathrm{kpc}$ (Kent, Dame \& Fazio 1991; Drimmel \& Spergel 2001; Narayan \& Jog 2002; López-Corredoira et al. 2002; Momany et al. 2006). The behaviour of this flare in the central discs of spiral galaxies has not been studied so well due to inherent difficulties in separating the several contributions to the observed counts or flux. López-Corredoira et al. (2004), for example, find that there is a deficit of stars compared to the predictions of a pure exponential law in the inner $4 \mathrm{kpc}$ of the Milky Way, which could be explained as being a flare which displaces the stars to higher heights above the plane as we move to the Galactic centre.

In this scenario, where on the one hand the mean disc $(z=0)$ can be displaced as much as $2 \mathrm{kpc}$ between the location of the maximum and the minimum amplitudes of the warp (Drimmel \& Spergel 2001; López-Corredoira et al. 2002; Momany et al. 2006), and on the other the scaleheight of the stars can show differences up to $50 \%$ of the value for $h_{\mathrm{z}}\left(R_{\odot}\right)$ in the range $5<R<10 \mathrm{kpc}$ (Alves 2000; López-Corredoira et al. 2002; Momany et al. 2006) to fit a global Galactic disc model that accounts for all these inhomogeneities is, at the very least, tricky. Because of this, the results in the Galactic model parameters might depend on the sample of Galactic coordinates used, as the combined effect of the warp and flare will be different at different directions in the Galaxy and hence along different lines of sight.

There is an additional reason for the differences between the numerical values of a given Galactic model parameter estimated in different directions into the Galaxy, mainly at larger galactocentric distances. These are the observed overdense regions with respect to an axisymmetric halo, for which two competing scenarios have been proposed: the first one is concerned with the triaxiality of the halo (Newberg \& Yanny 2006; Xu, Deng $\& \mathrm{Hu} 2006$; Jurić et al. 2008) whereas the second one is related to the remnants of some historical merger events (Wyse \& Gilmore 2005).

In this paper, we derive the structural parameters of two discs and the halo of the Galaxy from very recent SDSS data to observe possible changes in the parameters with the Galactic longitude. We used about 1.27-million stars in 36 high-latitude fields, which cover the whole longitude interval $\left(0 \leq l \leq 360^{\circ}\right)$, and we evaluated their absolute magnitudes by means of recent procedures which provide accurate distance determination. In Section 2 we describe the SDSS data, as well as the density laws, absolute magnitudes, distances, and density functions employed in the analysis. Estimation of the Galactic model parameters and their dependence with the Galactic longitude is given in Section 3. Finally, our main results are discussed and summarised in Sections 4 and 5, respectively.

\section{SDSS}

The SDSS is a large, international collaboration project set up to survey 10000 square-degrees of sky in five optical passbands and to obtain spectra of one million galaxies,
100000 quasars, and tens of thousands of Galactic stars. The data are being taken with a dedicated 2.5-m telescope located at Apache Point Observatory (APO), New Mexico. The telescope has two instruments: a CCD camera with $302048 \times 2048$ CCDs in the focal plane and two 320fibre double spectrographs. The imaging data are tied to a network of brighter astrometric standards (which would be saturated in the main imaging data) through a set of 22 smaller CCDs in the focal plane of the imaging camera. An 0.5-m telescope at APO will be used to tie the imaging data to brighter photometric standards.

The SDSS obtains images almost simultaneously in five broad bands $(u, g, r, i \text { and } z)^{1}$ centred at $3551,4686,6166$, 7480 and $8932 \AA$, respectively (Fukugita et al. 1996). The imaging data are automatically processed through a series of software pipelines which find and measure objects and provide photometric and astrometric calibrations to produce a catalogue of objects with calibrated magnitudes, positions and structure information. The photometric pipeline (Lupton et al. 2001) detects the objects, matches the data from the five filters, and measures instrumental fluxes, positions, and shape parameters (which allows the classification of objects as 'point source', i.e. compatible with the point spread function, or 'extended'). The photometric calibration is accurate to roughly $2 \% \mathrm{rms}$ in the $g, r$ and $i$ bands, and 3\% in $u$ and $z$, as determined by the constancy of stellar population colours (Ivezic et al. 2004; Blanton et al. 2005), while the astrometric calibration precision is better than 0.1 arcsec rms per coordinate (Pier et al. 2003). The Data Release 5 (DR5) imaging catalogue covers $8000 \mathrm{deg}^{2}$ (Adelman-McCarthy et al. 2007) with a detection repeatability complete at a $95-\%$ level for point sources brighter than the limiting apparent magnitudes of 22.0, 22.2, 22.2, 21.3 and $20.5 \mathrm{mag}$ for $u, g, r, i$ and $z$, respectively. The data are saturated at about $14 \mathrm{mag}$ in $g, r$ and $i$ and about $12 \mathrm{mag}$ in $u$ and $z$.

\subsection{Observational Data and Reduction}

The data used in this work were taken from SDSS (DR5) WEB server ${ }^{2}$ for 36 high-latitude fields $\left(60 \leq b \leq 65^{\circ}\right)$ covering different Galactic longitude intervals $\left(0 \leq l \leq 360^{\circ}\right)$. SDSS magnitudes $u, g, r, i$, and $z$ were used in a total number of 2164680 stars in 36 fields equal in size ( $831 \mathrm{deg}^{2}$ in total). Although the fields are equal in size $\left(23.08 \mathrm{deg}^{2}\right)$, their surface densities (number of stars per square-degree) are not the same, following a specific trend with Galactic longitude (Figure 1). This is the first clue to the dependence of the Galactic model parameters on Galactic longitude. Owing to the SDSS observing strategy, stars brighter than $g_{0}=14$ mag are saturated, and star counts are not be complete for magnitudes fainter than $g_{0}=22.2 \mathrm{mag}$. Hence, our work is restricted to the magnitude range $15<g_{0} \leq 22$ for the evaluation of the Galactic model parameters.

\footnotetext{
${ }^{1}$ Magnitudes in this paper are quoted in the ugriz system to differentiate them from the former one, $u^{\prime} g^{\prime} r^{\prime} i^{\prime} z^{\prime}$.

${ }^{2}$ http: //www.sdss.org/dr5/access/index.html
} 


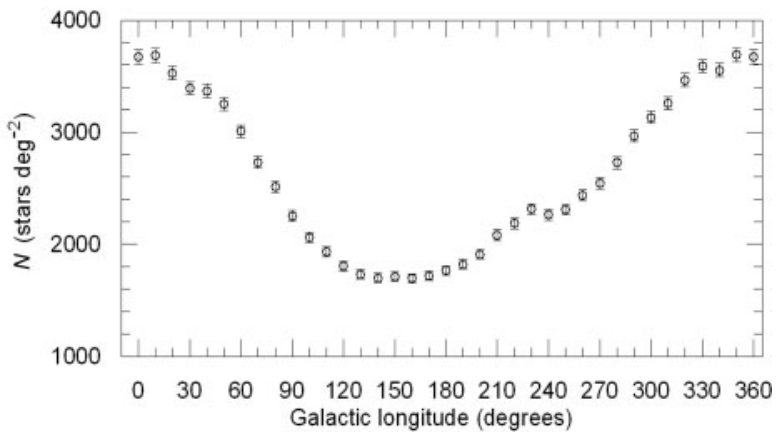

Figure 1 Star counts at $b=+62.5^{\circ}$ for the 36 fields available in the DR5.

The $E(B-V)$ colour excess was evaluated individually for each subsample source by using the maps of Schlegel, Finkbeiner \& Davis (1998) through SDSS query server and this was reduced to total absorption $A_{\mathrm{V}}$ by

$$
A_{\mathrm{V}}=3.1 E(B-V) .
$$

In order to determine total absorptions, $A_{\mathrm{m}}$, for the $S D S S$ bands, $A_{\mathrm{m}} / A_{\mathrm{V}}$ data given by Fan (1999), i.e. 1.593, 1.199 , $0.858,0.639$ and 0.459 for $m=u, g, r, i$ and $z$, respectively, were used. Thus, the de-reddened magnitudes, with subscript 0 , are

$$
\begin{aligned}
& u_{0}=u-A_{\mathrm{u}}, \\
& g_{0}=g-A_{\mathrm{g}}, \\
& r_{0}=r-A_{\mathrm{r}}, \\
& i_{0}=i-A_{\mathrm{i}}, \\
& z_{0}=z-A_{\mathrm{z}} .
\end{aligned}
$$

The total absorptions $A_{\mathrm{m}}$ are avaliable in the SDSS query server.

All the colours and magnitudes mentioned hereafter will be de-reddened ones. Given that the location of the vast majority of our targets are at distances larger than $0.4 \mathrm{kpc}$, it seems appropriate to apply the full extinction from the maps. Actually, when we combine the distance $r=0.4 \mathrm{kpc}$ (distance $0.35 \mathrm{kpc}$ from the Galactic plane) with the scaleheight of the dust, (Marshall et al. 2006, $H=125 \mathrm{pc}$ ), we find that the total extinction is reduced to $6 \%$ of the value at the Galactic plane at the nearest distance of stars in our work.

According to Chen et al. (2001), the distribution of stars in the $g_{0} /(g-r)_{0}$ colour-magnitude diagram (CMD) can be classified as follows: blue stars in the range magnitude $15<g_{0}<18$ are dominated by thick-disc stars with a turn-off at $(g-r)_{0} \approx 0.33 \mathrm{mag}$, while Galactic halo stars become significant for $g_{0}>18 \mathrm{mag}$, with a turn-off at $(g-r)_{0} \approx 0.2 \mathrm{mag}$; red stars, $(g-r)_{0} \geq 1.3 \mathrm{mag}$, are dominated by thin disc stars at all apparent magnitudes. The CMD, $g_{0} /(g-r)_{0}$, in Figure 2 shows the mentioned populations and, spectral types and absolutes magnitude of stars of these populations.

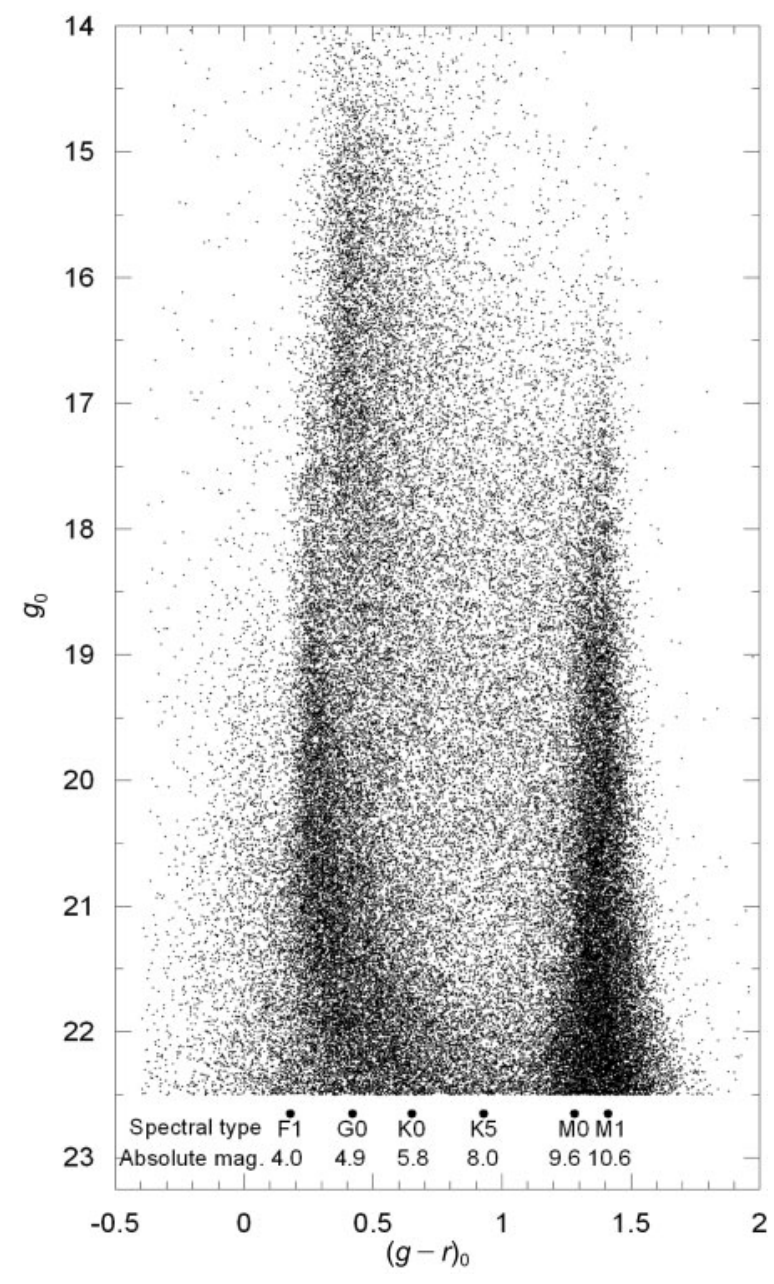

Figure 2 Colour magnitude diagram for the star sample. Spectral types and absolute magnitudes are indicated in the horizontal axis. Then thin disc stars are dominant at the red peak whereas the thick disc $\left(14<g_{0} \leq 18\right)$ and the halo $\left(g_{0}>18\right)$ stars are concentrated at the blue peak.

However, the CMDs and the two-colour diagrams for all objects (not presented here) indicate that the stellar distributions are contaminated by extragalactic objects as claimed by Chen et al. (2001). The star/extragalactic object separation is based on the 'stellarity parameter' as returned from the SExTRACTOR routines (Bertin \& Arnouts 1996). This parameter has a value between 0 (high extended) and 1 (point source). The separation works very well to classify a point source with a value greater than 0.8. Needless to say, this separation depends strongly on seeing and sky brightness. We also applied the 'locus-projection' method of Juric et al. (2008) in order to remove hot white dwarfs, low-redshift quasars, and white/ red-dwarf unresolved binaries from our sample. Briefly, this procedure consists of rejecting objects at distances larger than $0.3 \mathrm{mag}$ from the stellar locus (Figure 3).

\subsection{Density Laws}

In this work we adopted the density laws of the Basle group (Buser et al. 1998, 1999). Disc structures are usually 


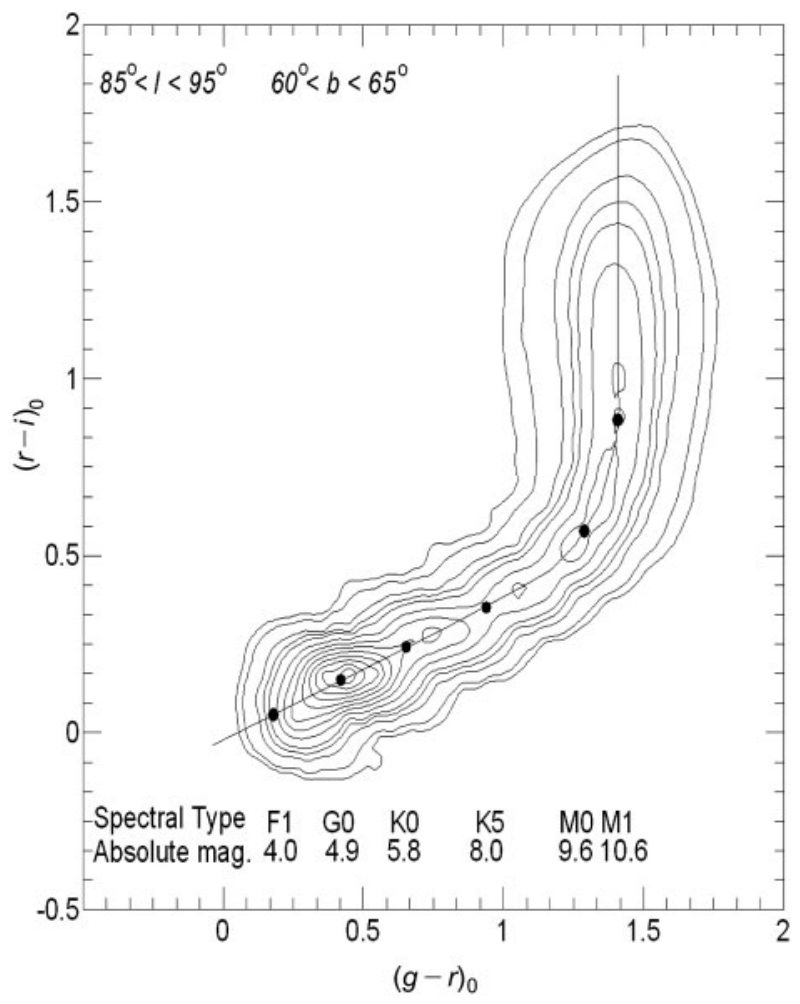

Figure $3(g-r)_{0} /(r-i)_{0}$ two colour diagram for the field centred at $l=90^{\circ}$. Isodensity contours show the position of stars at distance $d<0.3 \mathrm{mag}$ from the stellar locus, adopted from Jurić et al. (2008). Black circles represent the dwarf stars with spectral types and absolute magnitudes stated in two lines below the diagram.

parameterized in cylindrical coordinates by radial and vertical exponentials:

$$
D_{i}(R, z)=n_{i} \exp \left(-\frac{|z|}{h_{\mathrm{z}, i}}\right) \exp \left[-\frac{\left(R-R_{0}\right)}{h_{i}}\right]
$$

where $z=z_{\odot}+r \sin (b), r$ is the distance to the object from the Sun, $b$ the Galactic latitude, $z_{\odot}$ the vertical distance of the Sun from the Galactic plane (Jurić et al. 2008, $24 \mathrm{pc}), R$ the projection of the galactocentric distance on the Galactic plane, $R_{0}$ the solar distance from the Galactic centre (Reid 1993, $8 \mathrm{kpc}$ ), $h_{\mathrm{z}, i}$ and $h_{i}$ are the scaleheight and scalelength, respectively, and $n_{i}$ is the normalized density at the solar radius. The suffix $i$ takes the values 1 and 2 as long as the thin and thick discs are considered. As this study focuses on the dependence of the scaleheight and solar normalization on the Galactic longitude, we fixed their scalelengths to 2.4 and $3.5 \mathrm{kpc}$ for thin and thick discs, respectively, according to Jurić et al. (2008).

The density law for the spheroid component is parameterized in different forms. The most common is the de Vaucouleurs (1948) spheroid used to describe the surface brightness profile of elliptical galaxies. This law has been deprojected into three dimensions by Young (1976) as

$$
D_{\mathrm{s}}(R)=\frac{n_{\mathrm{S}} \exp \left[-7.669\left(R / R_{\mathrm{e}}\right)^{1 / 4}\right]}{\left(R / R_{\mathrm{e}}\right)^{7 / 8}},
$$

where $R$ is the (uncorrected) Galactocentric distance in spherical coordinates, $R_{\mathrm{e}}$ is the effective radius and $n_{\mathrm{S}}$ is the normalized local density. $R$ has to be corrected for the axial ratio $\kappa=c / a$,

$$
\begin{aligned}
R & =\sqrt{x^{2}+\left(\frac{z}{\kappa}\right)^{2}}, \\
z & =z_{\odot}+r \sin b \\
x & =\sqrt{R_{0}^{2}+\left(\frac{z}{\tan b}\right)^{2}-2 R_{0}\left(\frac{z}{\tan b}\right) \cos l},
\end{aligned}
$$

with $r$ the distance along the line of sight and, $b$ and $l$ the Galactic latitude and longitude respectively, for the field under investigation. The form used by Basle group (Fenkart 1989a,b,c,d) is independent of effective radius but is dependent on the distance from the Sun to the Galactic centre,

$$
D_{\mathrm{s}}(R)=\frac{n_{\mathrm{s}} \exp \left\{10.093\left[1-\left(R / R_{0}\right)^{1 / 4}\right]\right\}}{\left(R / R_{0}\right)^{7 / 8}} .
$$

\subsection{Absolute Magnitudes, Distances, and Density Functions}

Absolute magnitudes were determined by two different procedures. For absolute magnitudes $4<M_{\mathrm{g}} \leq 8$ we used the procedure of Karaali, Bilir \& Tunçel (2005, hereafter $\mathrm{KBT}$ ), whereas for $8<M_{\mathrm{g}} \leq 10$ we adopted the procedure of Bilir, Karaali \& Tunçel (2005, hereafter BKT). The cited absolute magnitude intervals correspond to the spectral type intervals F0-K5 and K5-M0 respectively. In the procedure of KBT, the absolute magnitude offset from the Hyades main sequence, $\Delta M_{\mathrm{g}}^{\mathrm{H}}$, is given as a function of both $(g-r)_{0}$ colour and $\delta_{0.43} U V$-excess, as follows:

$$
\Delta M_{\mathrm{g}}^{\mathrm{H}}=c_{3} \delta_{0.43}^{3}+c_{2} \delta_{0.43}^{2}+c_{1} \delta_{0.43}+c_{0},
$$

where $\delta_{0.43}$ is the $U V$-excess of a star relative to a Hyades star of the colour-index $(g-r)_{0}=0.43$ which corresponds to $\delta_{0.6}$ and which is determined by the colour equations between $U B V$ and $S D S S$ photometry (KBT). The coefficients $c_{i}(i=0,1,2,3)$ are functions of $(g-r)_{0}$ colour and they are adopted from the work of KBT, and where $\Delta M_{\mathrm{g}}^{\mathrm{H}}$ is defined as the difference in absolute magnitudes of a program star and a Hyades star of the same $(g-r)_{0}$ colour:

$$
\Delta M_{\mathrm{g}}^{\mathrm{H}}=M_{\mathrm{g}}^{*}-M_{\mathrm{g}}^{\mathrm{H}},
$$

The absolute magnitude for a Hyades star can be evaluated from the Hyades sequence, normalized by KBT (Equation 15 of KBT). This procedure is the one used in the work of Ak et al. (2007b), and has two main advantages: 1) there is no need to separate the stars into different populations and 2) the absolute magnitude of a star is determined from its $U V$-excess individually, which provides more accurate absolute magnitudes compared with the procedure 'in-situ', where a specific CMD is used for all stars of the same population. When one uses the last 
two equations (Equations 13 and 14) and Equation 15 of KBT simultaneously, it gets the absolute magnitude $M_{\mathrm{g}}^{*}$ of a star.

The procedure of KBT was defined only for the colours $0.09<(g-r)_{0} \leq 0.93$, which corresponds to absolute magnitudes $4<M_{\mathrm{g}} \leq 8$. Hence, for the absolute magnitudes $8<M_{\mathrm{g}} \leq 10$ we used the equation of BKT, which provides absolute magnitudes for late-type dwarfs:

$$
M_{\mathrm{g}}=5.791(g-r)_{0}+1.242(r-i)_{0}+1.412 .
$$

Stars with faint absolute magnitudes are very useful, since they provide estimates for the space densities at short distances relative to the Sun which combine the space densities for absolutely bright stars at large distances, and the local densities of Hipparcos (Jahreiss \& Wielen 1997). Thus, we have a sample of stars with absolute magnitudes $4<M_{\mathrm{g}} \leq 10$ which enables us to evaluate space density functions in the heliocentric distances interval $0.4<r \leq 25 \mathrm{kpc}$, corresponding to a range of distances of $0.4<z \leq 21 \mathrm{kpc}$ from the Galactic plane. This interval is large enough to estimate a set of Galactic model parameters and test their change with the Galactic longitude. The absolute magnitudes in question and the corresponding spectral types (from early $\mathrm{F}$ type to early $\mathrm{M}$ type) for the locus points in the $(g-r)_{0} /(r-i)_{0}$ two-colour diagram is shown in Figure 3 for the field centred at $l=90^{\circ}$ as an example. The local space density in the interval $4<M_{\mathrm{g}} \leq 10$ is flat and it attributes a mean value of logarithmic space density $D^{*}=7.49$.

In a conical magnitude-limited volume, the distance to which intrinsically bright stars are visible is larger than the distance to which intrinsically faint stars are visible. The effect of this is that brighter stars are statistically overrepresented and the derived absolute magnitudes are too faint. This effect, known as Malmquist bias (Malmquist 1920), was formalized into the general formula:

$$
M_{\mathrm{g}}=M_{0}-\sigma^{2} \frac{\mathrm{d} \log A(g)}{\mathrm{d} g} \text {, }
$$

where $M_{\mathrm{g}}$ is the assumed absolute magnitude, $M_{0}$ is the absolute magnitude calculated for any star using KBT calibration, $\sigma$ is the dispersion of the KBT or BKT calibration, and $A(g)$ is the differential counts evaluated at the apparent magnitude $g_{0}$ of any star. The dispersion in absolute magnitude calibration of KBT and BKT is around $0.25 \mathrm{mag}$, corresponding an error about $10 \%$ in photometric distance. We divided stars into the absolute magnitude intervals $(4,5],(5,6],(6,7],(7,8],(8,9)$ and $(9,10]$, and we applied the Malmquist bias to stars in each interval separately. This approach provides (relative) uniform space densities which is the essential Malmquist bias. Thus, the corrections applied to the absolute magnitudes are $0.005,0.003$, $0.007,0.008,0.012$ and 0.012 for the absolute magnitude intervals cited above. The correction of the Malmquist bias was applied to the SDSS photometric data used in this work.

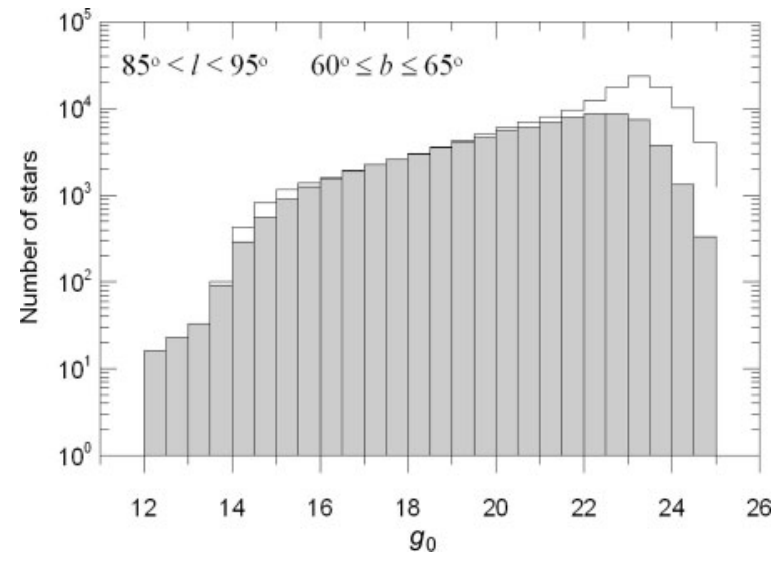

Figure 4 Apparent-magnitude histogram for point sources (white area) and for final stars sample (shaded area) for the field centred at $l=90^{\circ}$.

The combination of the absolute magnitude $M_{\mathrm{g}}$ and the apparent magnitude $g_{0}$ of a star gives its distance $r$ relative to the Sun, i.e.

$$
\left(g-M_{\mathrm{g}}\right)_{0}=5 \log r-5 .
$$

Gilmore, Wyse \& Jones (1995) quote an error of $\sim 0.2$ dex in the derivation of $[M / H]$ from the $U B V$ photometry for F/G stars which leads to a random uncertainty of $20 \%$ in the distance estimation. One expects larger distance errors for later spectral type stars. However, $(u-g)$ and $(g-r)$ colours are more accurate than the $(U-B)$ and $(B-V)$ colours which mitigates this excess error for K stars.

Logarithmic space densities $D^{*}=\log D+10$ have been evaluated for the combination of three population components (thin and thick discs and halo), for each field where $D=N / \Delta V_{1,2} ; \Delta V_{1,2}=(\pi / 180)^{2}(A / 3)\left(r_{2}^{3}-r_{1}^{3}\right)$; $A$ denotes the size of the field $\left(23.08 \mathrm{deg}^{2}\right) ; r_{1}$ and $r_{2}$ are the lower and upper limiting distances of the volume $\Delta V_{1,2} ; N$ is the number of stars per unit absolute magnitude; $r^{*}=\left[\left(r_{1}^{3}+r_{2}^{3}\right) / 2\right]^{1 / 3}$ is the centroid distance of the volume $\Delta V_{1,2}$; and $z^{*}=r^{*} \sin (b), b$ being the Galactic latitude of the field centre. The limiting distances of completeness, $z_{1}$, can be calculated from the following equations:

$$
\begin{aligned}
\left(g_{1}-M_{\mathrm{g}}\right)_{0} & =5 \log r_{1}-5, \\
z_{1} & =r_{1} \sin (b),
\end{aligned}
$$

where $g_{1}$ is the limiting apparent magnitude (15 and 22, for the bright and faint stars respectively, see Figure 4), $r_{1}$ is the limiting distance of completeness relative to the Sun, and $M_{\mathrm{g}}$ corresponds the absolute magnitude defining the interval $\left(M_{1}, M_{2}\right]$ where $\left(M_{1}, M_{2}\right]$ is $(4,5],(5,6]$, $(6,7],(7,8],(8,9]$ and $(9,10]$. For the limiting distance of completeness at short and large distances $M_{\mathrm{g}}$ assumes the bright and faint absolute magnitudes for each absolute magnitude interval. That is, the limiting distance of completeness is defined for each absolute magnitude to a limited range of spectral types. 


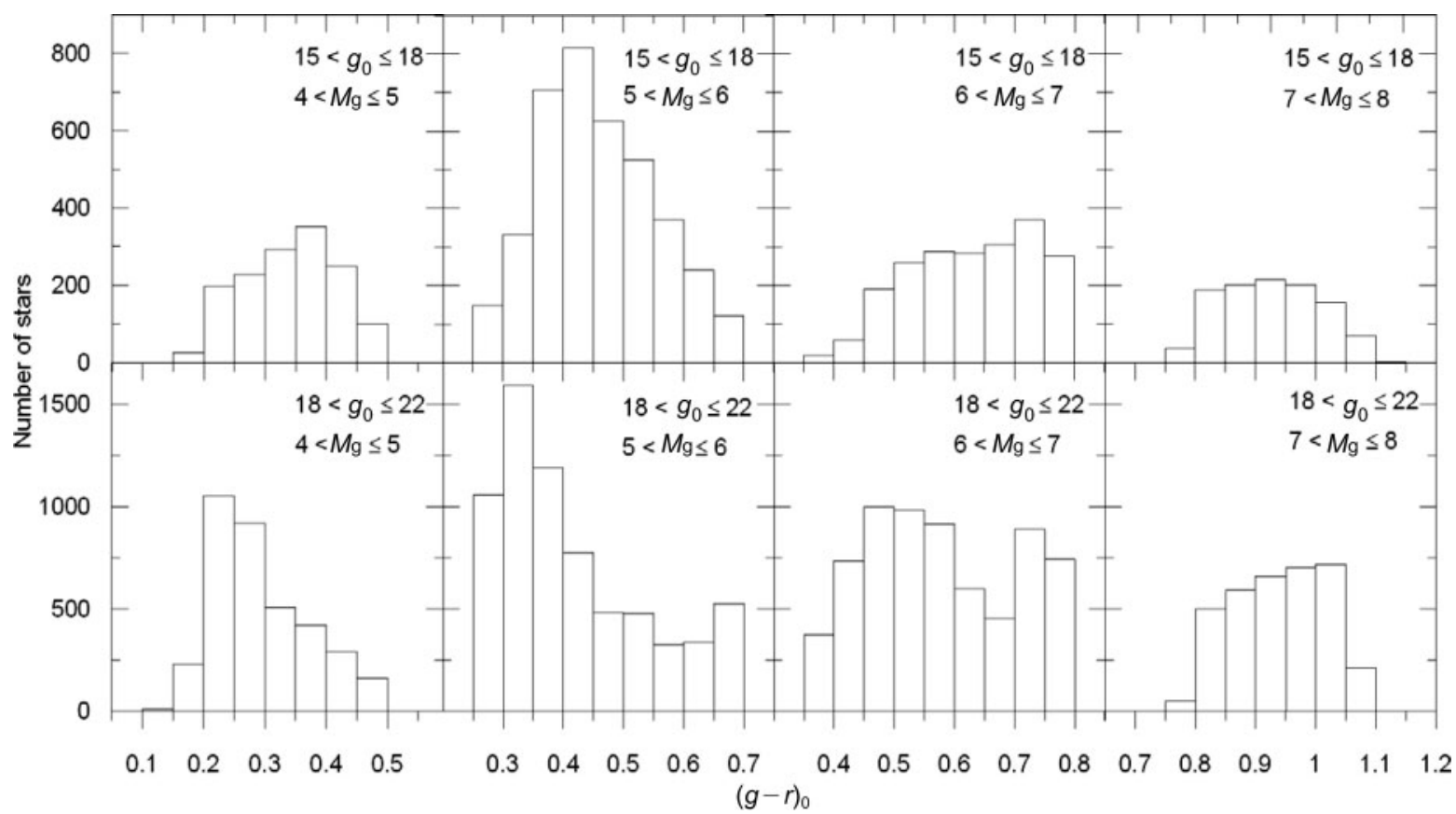

Figure 5 The $(g-r)_{0}$ colour distribution as a function of apparent and absolute magnitudes, for the field centred at $l=90^{\circ}$.

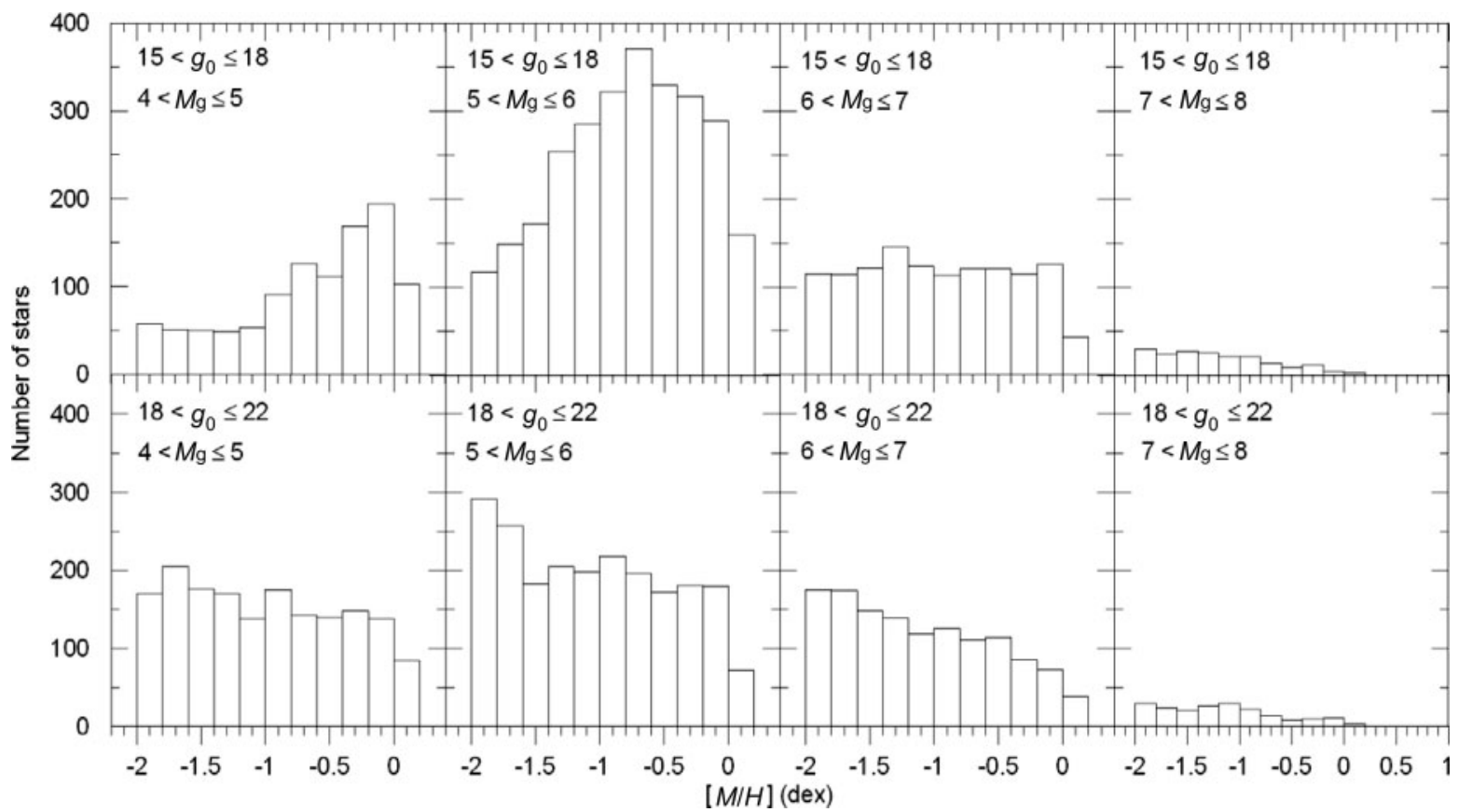

Figure 6 The metallicity distribution as a function of apparent and absolute magnitudes, for the field centred at $l=90^{\circ}$.

We present the distribution of $(g-r)_{0}$ colours and $[M / H]$ metallicities for stars in the field centred at $l=90^{\circ}$, as an example, to show the variation in these parameters as a function of apparent $g_{0}$ and absolute $M_{\mathrm{g}}$ magnitudes. Figure 5 shows how for the same apparent-magnitude interval the peaks of the histograms move to redder colours when one goes to fainter absolute magnitudes. This result is in agreement with the fact that stars closer to the Sun are late-type stars, i.e. thin-disc stars. Another result is that the peaks of the histograms for stars with fainter apparent magnitudes $\left(18<g_{0} \leq 22\right)$, but with the same absolute magnitudes as the brighter ones, occupy bluer colours. This confirms the suggestion of Chen et al. (2001), who demonstrated in their Figure 6 that apparently fainter stars $\left(g_{0}>18\right)$ are dominated by blue stars, i.e. halo stars.

Additionally, Figure 6 shows three peaks (at metalrich, intermediate and metal-poor parts) in the metallicity distribution. However, all of them are not conspicuous 
in the same panel, as they correspond to different absolute and apparent magnitude intervals. If we compare the upper and lower panels for stars with the same absolute magnitudes but different apparent magnitudes, we notice that the peaks shift to lower metallicities when one goes from relatively bright to faint apparent magnitude intervals. As different peaks mean different components of our Galaxy, i.e. thin and thick discs and halo, the result stated above confirms our previous finding (Karaali et al. 2007) that different populations are dominant at different absolute magnitudes. Most of the stars with fainter absolute magnitudes, $6<M_{\mathrm{g}} \leq 7$ for example, are metal rich stars (disc stars), whereas the absolutely brightest stars, i.e. $4<M_{\mathrm{g}} \leq 5$, have low metallicities, $[M / H]<-1 \mathrm{dex}$, and hence belong to the halo component of our Galaxy. As Carney (1979) quoted, the $U V$-excess at the red end is small, which limits the accuracy of the metallicity estimation (see Figure 16 in the Appendix). However, the general trend of metallicity distribution in Figure 6 does not give the indication of such an effect.

We acknowledge that in this work we have not applied any correction for binarity or giant/subgiant stars. However, most of the evolved stars are probably rejected automatically due to the limiting apparent magnitude at the bright end, i.e. $g_{0}=15$. We compared the number of giants and dwarfs with apparent magnitudes $g_{0}>15$ for a reliable confirmation of our argument. We adopted a mean absolute magnitude $M_{\mathrm{g}}=1.5$ for giants and we calculated their corresponding distance from the Galactic plane, $z=4.5 \mathrm{kpc}$, for $g_{0}=15$. We used the local space density $D^{*}=6.35$ and the scaleheight $(H=650 \mathrm{pc})$ for the thick disc dwarfs and we compared the corresponding space density with that of thick disc giants for local space density $D^{*}=5.58$ and the scaleheight $(H=585 \mathrm{pc})$ adopted from Bilir et al. (2006c). It turned out that the number of thick disc giants fainter than $g_{0}=15 \mathrm{mag}$ is less than $8 \%$ of the number of dwarfs. The number of halo giants relative to the number of halo dwarfs at distances $z \geq 4.5 \mathrm{kpc}$ is even less, i.e. $2 \%$. We used the selection criteria for metal-poor giants of Helmi et al. (2003) to estimate the cited number of halo giants. These authors define the location of the metal-poor giants by the following criteria: $r<19 \mathrm{mag}, 1.1 \leq(u-g)_{0} \leq 2.0$, $0.3 \leq(g-r)_{0} \leq 0.8, \quad-0.1<P_{1}<0.6, \quad|s|>m_{\mathrm{s}}+0.05$, where $P_{1}=0.910(u-g)_{0}+0.415(g-r)_{0}-1.28, \quad s=$ $-0.249 u+0.794 g-0.555 r+0.24$ and $m_{\mathrm{s}}=0.002$. When we apply these criteria to stars with $4<M_{\mathrm{g}} \leq 10$ we obtain 460 giants corresponding to $2 \%$ of the total number of stars $(N=19325)$ for the field $85<l<95^{\circ}$.

The fraction of binary stars ranges from 25 to $50 \%$ depending on the spectral types of stars. If we assume a binary fraction of $50 \%$ then the inferred scaleheight in a photometric parallax evaluation is approximately $80 \%$ of the actual value (Siegel et al. 2002). However, as we compare the scaleheight of stars of a specific population for fields in different directions of the Galaxy, we are interested only in the relative values of scaleheight but not their actual values. Hence, disregarding the binarity does not affect our results.

\section{Galactic Model Parameters}

\subsection{Estimation of the Galactic Model Parameters}

We estimated all the Galactic model parameters (the local space densities and scaleheights for the thin and thick discs, and the local space density and axial ratio for the halo) simultaneously, by fitting the space density functions derived from the observations (combined for the three population components) to a corresponding combination of the adopted population-specific analytical density laws. The absolutely faintest stars in this work provide space densities at short distances from the Galactic plane, $z \sim 0.4 \mathrm{kpc}$. Hence it is possible to have reliable extrapolation between them and the space density of Hipparcos in the solar neighborhood, $D^{*}=7.49$ in logarithmic form (Jahreiss \& Wielen 1997), corresponding to the mean of the local space densities for stars with $4<M_{\mathrm{g}} \leq 10$.

We used the classical $\chi^{2}$ method for the estimation of the Galactic model parameters, a method which is made in the studies of the Galactic structure that has been used in the determination of the most recent numerical values for the Galactic model parameters (Phleps et al. 2000, 2005; Chen et al. 2001; Siegel et al. 2002; Du et al. 2003, 2006; Jurić et al. 2008). The comparison of the logarithmic density functions derived from the observations and the analytical density laws are given in Figure 7, for four fields with Galactic longitudes $l=0,90,180$ and $270^{\circ}$. $\chi_{\min }^{2}$ shows almost a symmetrical distribution, as can be observed from Figure 8 which is given as an example. Hence, the errors of the Galactic model parameters could be estimated by changing a given model parameter until an increase or decrease by 1 was achieved (Phleps et al. 2000). Table 1 lists the Galactic model parameters for 36 SDSS high-latitude $\left(60 \leq b \leq 65^{\circ}\right)$ fields, resulting from the fits of the analytical density profiles. The columns indicate: Galactic longitude $(l)$, scaleheight of thin $\left(h_{\mathrm{z}, 1}\right)$ and thick discs $\left(h_{\mathrm{z}, 2}\right)$, local space density of the thick disc $\left(n_{2} / n_{1}\right)$ and the halo $\left(n_{3} / n_{1}\right)$ relative to the thin disc, axial ratio of the halo $(c / a)$ and star number density of the field $(N)$, reduced chi-square minimum $\left(\widetilde{\chi}_{\min }^{2}\right)$, and the corresponding probability. The $\tilde{\chi}_{\min }^{2}$ values are low, whereas the probabilities are rather high confirming the reality of the Galactic model parameters.

We also used a different procedure just to test any possible degeneracy in the estimation of the model parameters. First we estimated the local space density and the axial ratio for the halo by comparing the logarithmic space density function for $z>5 \mathrm{kpc}$ with the analytical density law of the halo (Equation 12). Then we omitted the space density of the halo, estimated by the corresponding density law, and compared the new density function for $z \leq 5 \mathrm{kpc}$ with the combined density laws of thin and thick discs (Figure 9). This procedure provides Galactic model parameters for thin and thick discs independent of the model parameters of the halo. The result of the 


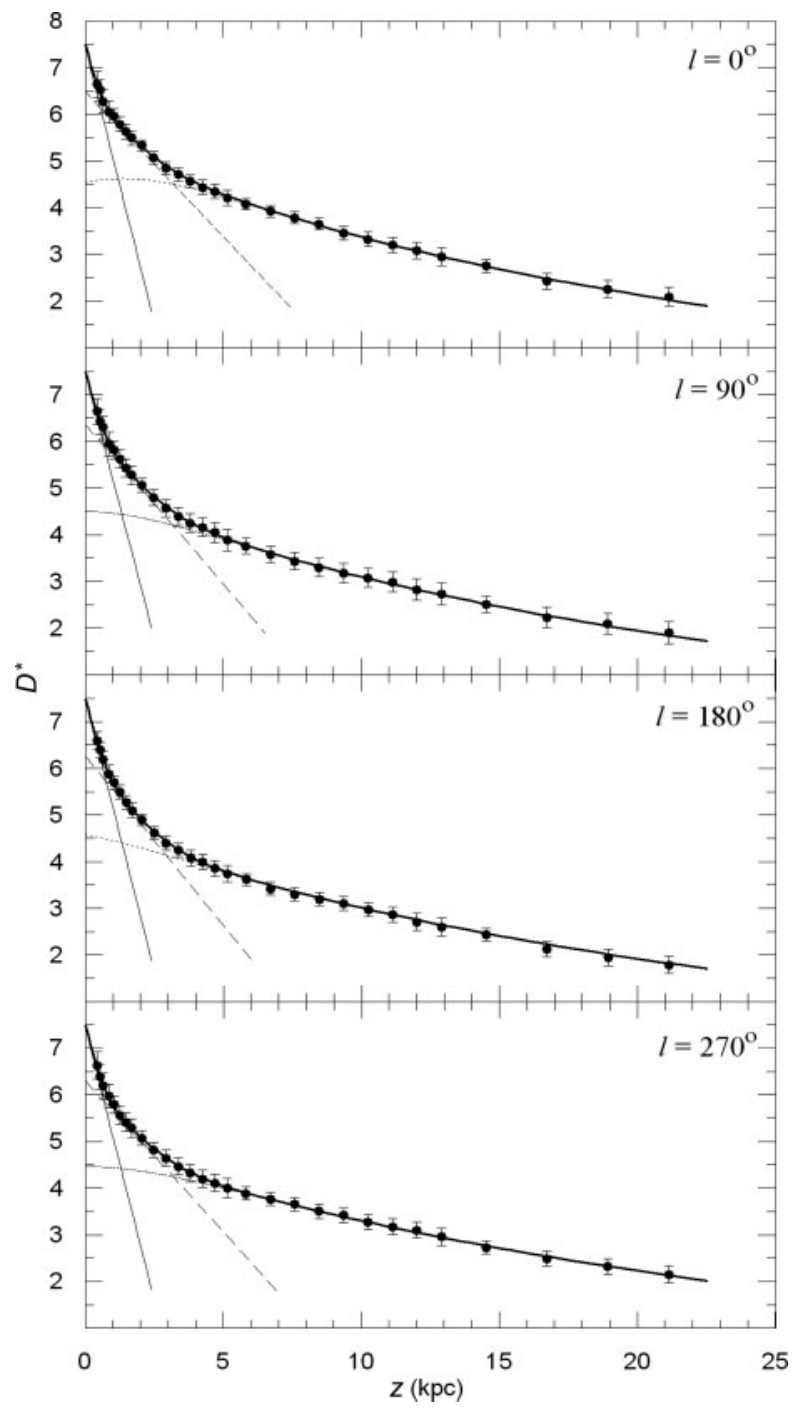

Figure 7 Observed (symbols) and evaluated (thick solid lines) space density functions combined for stars of all three population components: thin disc (thin solid line), thick disc (dashed line) and halo (dotted line), at four different Galactic longitudes.

application of the two different procedures shows that the corresponding Galactic model parameters for a specific population are either identical or they differ only by a negligible amount. Hence, we may argue that no degeneracy exists in the estimated parameters.

A similar procedure is applied to the thin and thick discs. We estimated the solar normalisations and the scaleheights of thin and thick discs simultaneously by the space density function for $0.4<z \leq 5 \mathrm{kpc}$ and compared the model parameters of the thick disc with the corresponding ones estimated by the space density function for $1.5<z \leq 5 \mathrm{kpc}$, where the thick disc is dominant. Since no significant differences could be observed between the compared parameters, we concluded that no degeneracy exists between the two discs either.

Additionally, we plotted the relative local space density of the thick disc versus its scaleheight, and the relative local space density of the halo versus its axial ratio, for four fields with Galactic longitudes $l=0,90,180$ and $270^{\circ}$
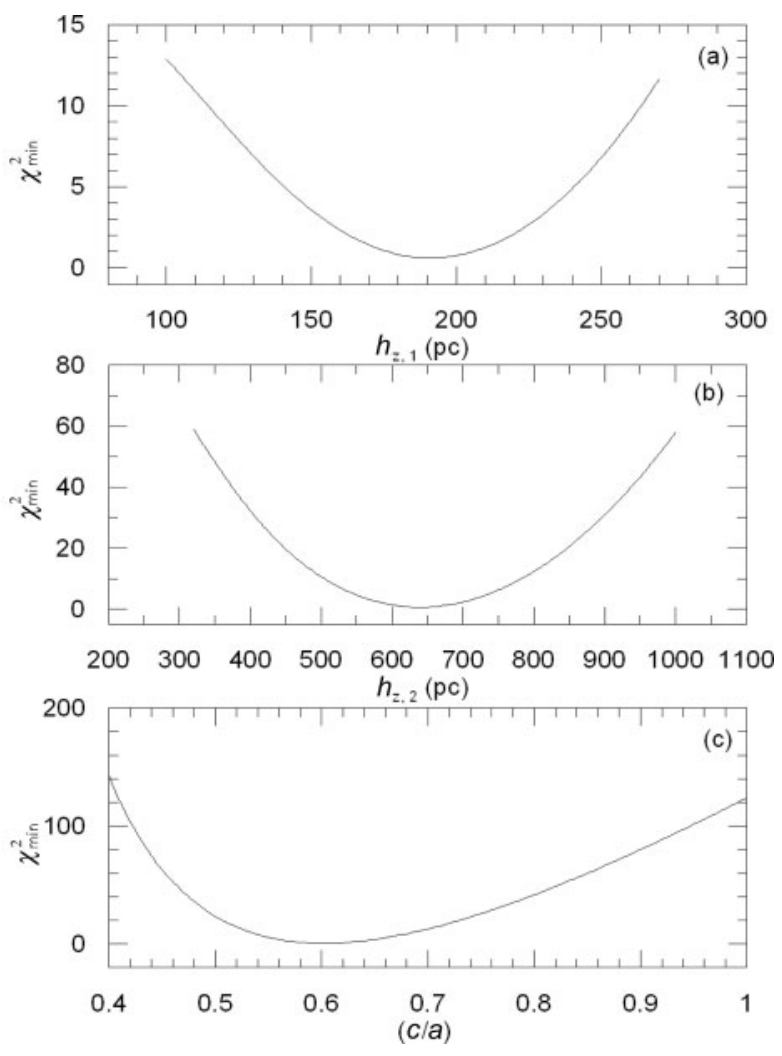

Figure 8 The variation of $\chi_{\min }^{2}$ with three Galactic model parameters. (a) with scaleheight of the thin disc, (b) with scaleheight of the thick disc, and (c) with axial ratio of the halo. The figures in panels (a) and (b) are symmetric and the one in panel (c) is slightly skewed to the right.

in Figure 10 to test the same problem. The contours correspond to the same $\chi^{2}$ for $\sigma, 2 \sigma$ and $3 \sigma$, where $\sigma$ is the standard deviation. In each panel, the cross shows the position for the minimum $\chi^{2}$, which defines the Galactic model parameters on the axes with accuracy.

\subsection{Dependence of the Galactic Model Parameters on Galactic Longitude}

Table 1 shows that the scaleheights of the thin and thick discs, as well as the relative local space densities of the thick disc and halo, even the axial ratio of the halo are not the same for 36 fields. That is, these Galactic model parameters change as a function of the Galactic longitude.

Figure 11 shows the variation in the scaleheight of the thin disc $\left(h_{\mathrm{z}}\right)$ with the Galactic longitude $(l)$. The global distribution of $h_{\mathrm{z}}$ has a maximum at $l \approx 190^{\circ}$, almost in the direction the anti-Galactic centre, whereas the minimum corresponds to the fields in the Galactic centre direction. However one can separate the bins into several sub-samples with segments of different slopes. Additionally, the segments corresponding to the fields with Galactic longitudes less than $150^{\circ}$ have negative slopes whereas the slopes of the ones with longitudes greater than $150^{\circ}$ are positive.

The trend of the scaleheight of the thick disc is different than the one the thin disc (Figure 12). The maximum and minimum of the scaleheight for the global distribution 

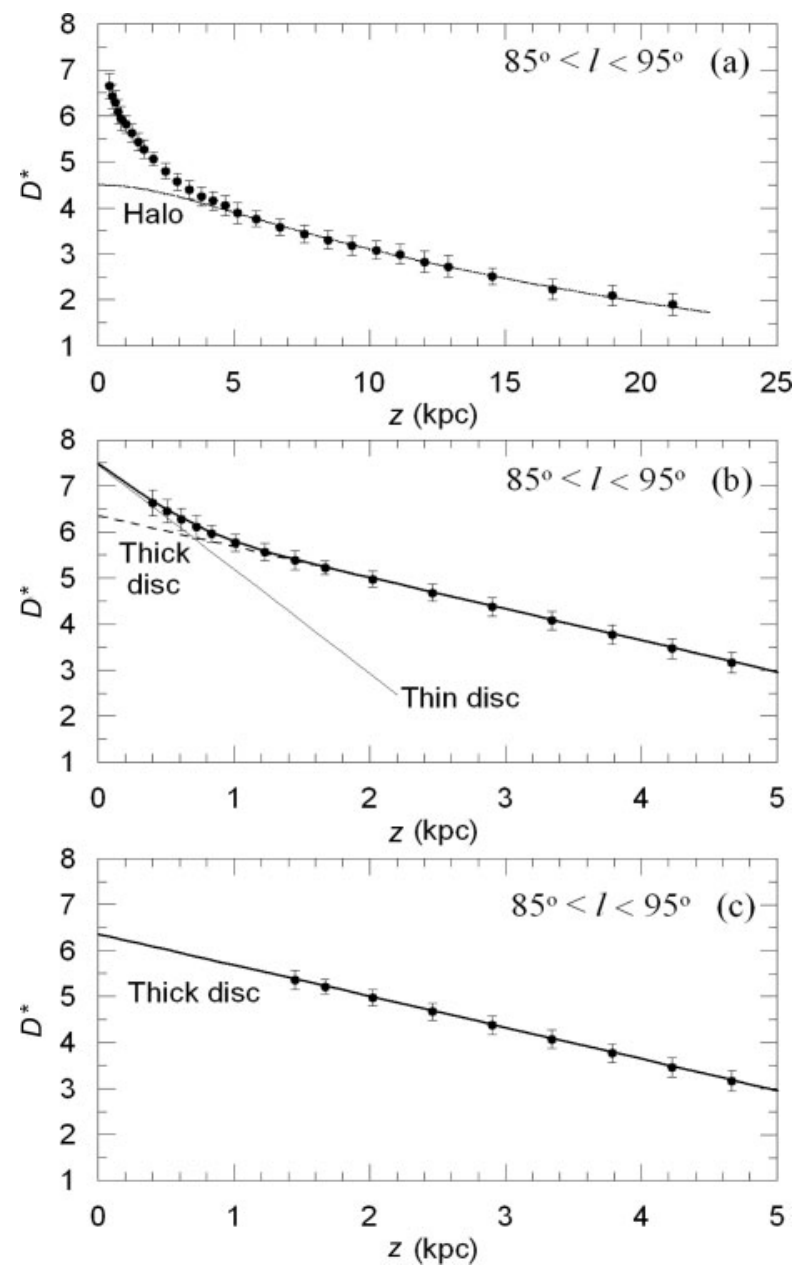

Figure 9 An alternative procedure for the estimation of the Galactic model parameters, for the field centred at $l=90^{\circ}$ as an example. In panel (a), the space densities based on the observational data for distances larger than $z=5 \mathrm{kpc}$ are compared with the analytical density law of the halo. Thus, only the local space density and the axial ratio of the halo are estimated independent of the Galactic model parameters of the thin and thick discs. In panel (b), the density of the halo estimated in panel (a) is omitted, and the density function of the remaining data for $z<5 \mathrm{kpc}$ is compared with the combined analytical density laws of the thin and thick discs, which provide local space densities and scaleheights for the thin and thick discs. Finally, in panel (c) only the density function for $1.5<z \leq 5 \mathrm{kpc}$, where the thick disc is dominant, is compared with the analytical density law of the thick disc. Thus, we estimated the model parameters of the thick disc individually. The Galactic model parameters estimated by this procedure are in agreement with the ones estimated by comparison of the combined observational based density function with the combined analytical density laws for three populations. This agreement excludes any possible degeneracy between halo and discs, and between two discs.

are $l \approx 290^{\circ}$ and $l \approx 140^{\circ}$, respectively. The slopes of the segments in this figure are also different from those in Figure 11 for the same longitude set. The error bars are also larger than those for the scaleheights of the thin disc. Different trends for the relative local space densities of the thick disc $\left(n_{2} / n_{1}\right)$ and halo $\left(n_{3} / n_{1}\right)$, and the axial ratio of the halo $(c / a)$ can be also observed in Figures 12 and 13. For example, the minimum of the global distribution of $\left(n_{2} / n_{1}\right)$ lies within $180 \leq l \leq 200^{\circ}$ whereas the minimum of $\left(n_{3} / n_{1}\right)$ lies in an interval with larger Galactic longitudes, $270 \leq l \leq 290^{\circ}$. We should note that one can also observe segments with different slopes in the distributions of the relative local space densities for the thick disc and halo, and of the axial ratio for the halo.

Apart from the variation of the Galactic model parameters with the Galactic longitude, one can say something about the correlation between the estimated parameters. Figure 12 shows that the scaleheight of the thick disc is an increasing function of Galactic longitude in an interval where the local space density of the thick disc is a decreasing function and vice versa. The same argument holds for the axis ratio and the local space density of the halo (Figure 13). Although there is a degeneracy between the scaleheight and the local space density of the thick disc, and between the axial ratio and the local space density of the halo (Figure 10), the mentioned correlations are real, since the corresponding reduced $\tilde{\chi}_{\min }^{2}$ are rather low (Table 1). These correlations were also cited in Buser et al. $(1998,1999)$ where seven fields in different directions of the Galaxy were investigated with $R G U$ photometry.

Table 2 gives the range, mean and standard deviations (columns 2, 3 and 4 respectively) of the Galactic model parameters as a function of Galactic longitude. The mean of the scaleheight of the thin disc is (at least) about $30 \%$ less than the one that appeared in the literature (Ojha et al. 1999; Buser et al. 1998, 1999; Karaali et al. 2004). For the thick disc the scaleheight and the relative local space density are close to the ones that appeared in the literature in recent years, also obtained from SDSS data. However, the upper limit of the space density is a bit higher than that cited until now (Chen et al. 2001; Siegel et al. 2002). The axial ratio and the relative local space density of the halo are rather close to the corresponding ones cited by many authors (Robin et al. 1996, 2000; Chen et al. 2001; Siegel et al. 2002; Karaali et al. 2004; Bilir et al. 2006b).

\section{Discussion}

\subsection{Evolution of the Concept of Galactic Model Parameters}

Galactic researchers have been working on the modelling of our Galaxy for about 25 years. The Galactic model parameters for the discs and halo have been refined since this epoch. There is a concensus about the idea that the most refined parameters are those of the thick disc. Actually, the scaleheight of the thick disc decreased from the original value of Gilmore \& Reid (1983), $1.45 \mathrm{kpc}$, to the recent one, $0.65 \mathrm{kpc}$ whereas the solar normalization increased from 2 to $6-10 \%$ (Siegel et al. 2002). Despite the same density laws, different model parameters with large ranges have been cited by different researchers (see Table 1 of Karaali et al. 2004). However, one expects Galactic model parameters either with small errors or with a short range from the recent surveys such as SDSS, SEGUE, 2MASS, DENIS, and UKIDSS which provide accurate 

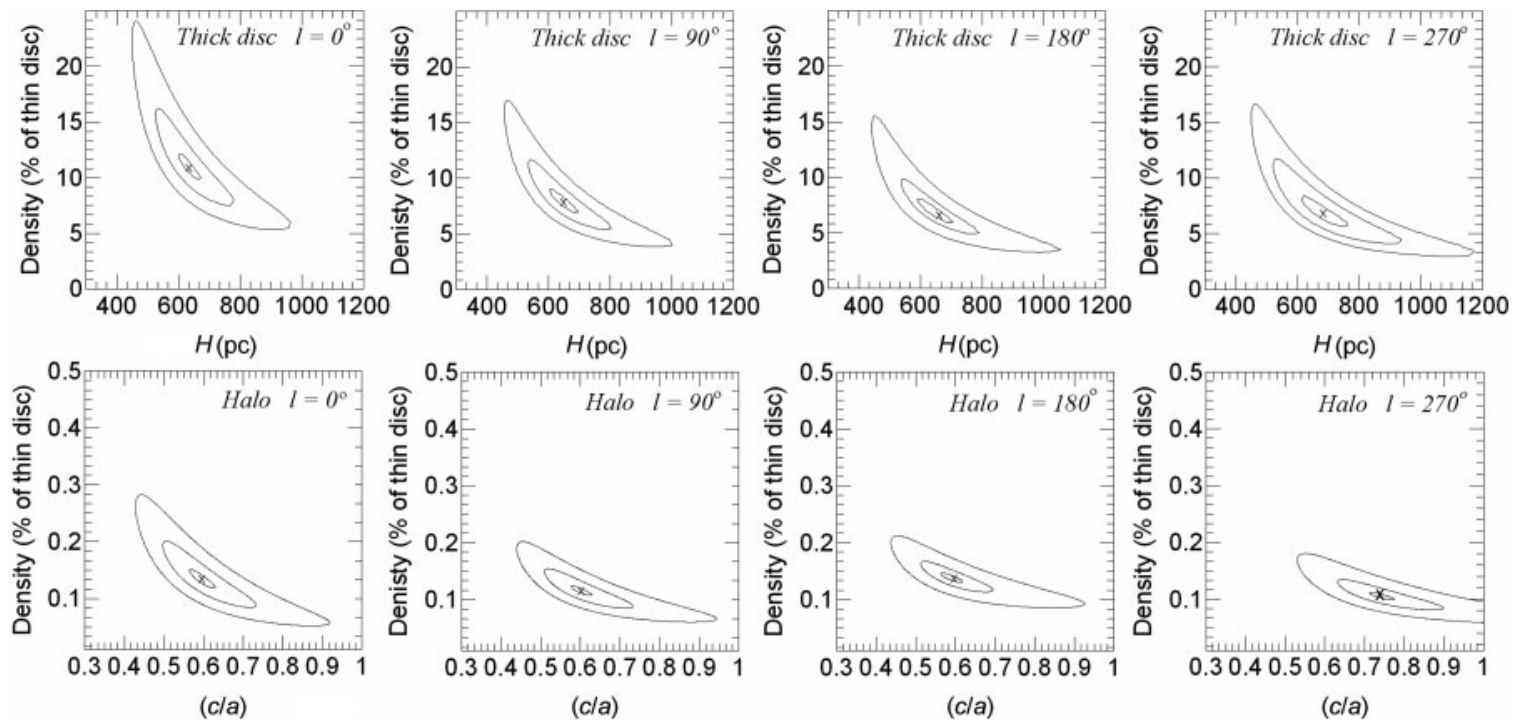

Figure 10 Contours of equal $\chi^{2}$ obtained for different values of scaleheight and local density for thick disc, and axial ratio and local density for halo for the fields with Galactic longitudes of $0,90,180$ and $270^{\circ}$. The cross $(\times)$ shows the minimum $\chi^{2}$ value in each panel while the contours show the $1 \sigma, 2 \sigma$, and $3 \sigma$ confidence levels.

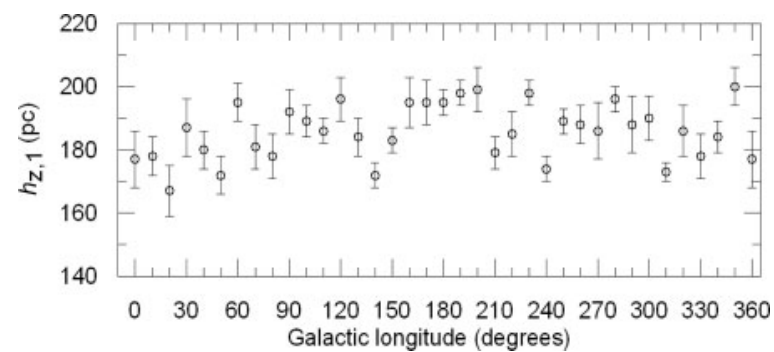

Figure 11 Variation in the scaleheight of the thin disc with the Galactic longitude (the Galactic latitudes of the fields lie within $\left.60 \leq b \leq 65^{\circ}\right)$.

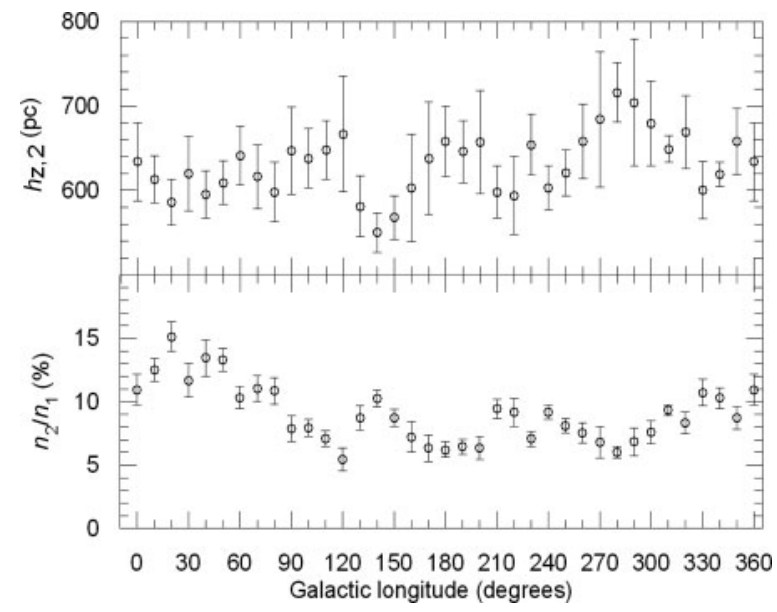

Figure 12 Variations in the scaleheight and relative space density of the thick disc with the Galactic longitude.

magnitudes and colours. It seems that we must approach the problem from a physical point of view.

We showed in Karaali et al. (2004) that the Galactic model parameters are absolute magnitude dependent. The errors for the model parameters estimated for a unit

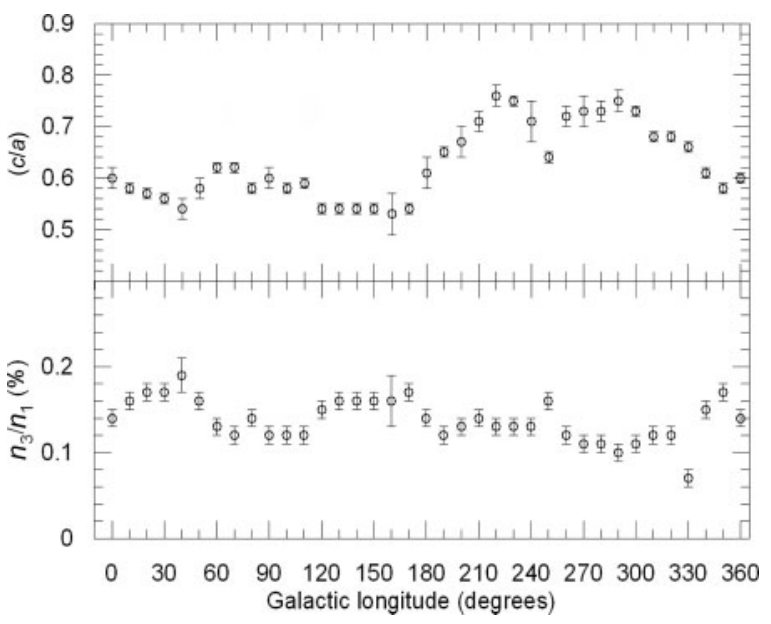

Figure 13 Variations in the axis ratio and relative space density of the halo with the Galactic longitude.

absolute magnitude interval are rather small, and the numerical values for a specific Galactic model parameter increases or decreases, depending on the parameter, with the absolute magnitude. For example, the range of the scaleheight of the thin disc is $264-334$ pc for the absolute magnitude interval $5<M_{\mathrm{g}} \leq 13$. A second example can be given for the thick disc. The range of the relative local space density of the thick disc for the absolute magnitude interval $5<M_{\mathrm{g}} \leq 9$ is $5.25-9.77 \%$, coincident with the classical value given in recent works, without paying attention to the dependence of this parameter on absolute magnitude. In other words, the large range of the Galactic model parameters is unavoidable in the procedure of star counts in which separation of stars into different absolute magnitude intervals is not regarded.

Different absolute magnitude intervals correspond to different spectral types and different populations. Stars with the brightest absolute magnitudes $\left(4<M_{\mathrm{g}} \leq 5\right)$, 
Table 2. Equations for the variation of the different Galactic model parameters

\begin{tabular}{lccc}
\hline Parameter & Range & Mean & $s$ \\
\hline$h_{\mathrm{z}, 1}(\mathrm{pc})$ & $167-200$ & 186 & 9 \\
$h_{\mathrm{z}, 2}(\mathrm{pc})$ & $550-716$ & 631 & 37 \\
$n_{2} / n_{1}(\%)$ & $5.45-15.14$ & 9.03 & 2.34 \\
$n_{3} / n_{1}(\%)$ & $0.07-0.19$ & 0.14 & 0.02 \\
$c / a$ & $0.53-0.76$ & 0.63 & 0.07 \\
\hline
\end{tabular}

intermediate $\left(5<M_{\mathrm{g}} \leq 8\right)$, and faintest absolute magnitudes $\left(8<M_{\mathrm{g}} \leq 10\right)$ are of spectral types $\mathrm{F}-\mathrm{G}, \mathrm{G}-\mathrm{K}$ and $\mathrm{K}-\mathrm{M}$, respectively (Figure 3 ). As claimed in our previous papers (Karaali et al. 2004; Bilir et al. 2006b) halo, thickdisc and thin-disc stars are dominant at these spectral-type intervals, respectively. Hence, different Galactic model parameters estimated for different absolute magnitude diagrams correspond to different populations and hence stellar ages.

\subsection{Interpretation of the Dependence of the Galactic Model Parameters on Galactic Longitude}

\subsubsection{Scenarios for the Asymmetric Structure of the Galaxy and its Confirmation by Star Counts}

The differences between the numerical values of a given Galactic model parameter estimated at different directions into the Galaxy (mainly at large galactocentric distances), could be explained by regions that are overdense with respect to an axisymmetric halo. Two competing scenarios have been proposed for this: the first one is concerned with the triaxiality of the halo (Newberg \& Yanny 2006; Xu et al. 2006; Jurić et al. 2008), whereas the second one is related to the remnants of some historical events (cf. Wyse \& Gilmore 2005). Although Newberg \& Yanny (2005) claimed that the thick disc was symmetric about the Galactic longitude $l=180^{\circ}$, Parker, Humphreys \& Larsen (2003) interpretered the excess in the numbers of blue- and 'intermediate' coloured stars above and below the Galactic plane in quadrant I as the asymmetric structure of the thick disc. Parker et al. (2003) propose similar scenarios for their observations: a) fossil remnant of a merger, b) a triaxial thick disc or halo and c) interaction of the thick-disc/inner-halo stars with the bar in the disc. The limiting magnitude in our work is much fainter $\left(g_{0}=22\right)$ than the limiting magnitude $(O=18)$ of the authors just mentioned. Hence, we thought we could obtain more reliable results, if we used a similar procedure.

We adopted the procedure of Newberg \& Yanny (2005) and we plotted the surface density (number of stars per square degree of a field) as a function of Galactic longitude, for different apparent-magnitude $(g)$ intervals, i.e. $(15,16],(16,17],(17,18],(18,19],(19$, 20], (20, 21], and (21, 22] (Figure 14). An asymmetric structure can be observed not only for the faint magnitudes which correspond to the halo stars, but also for the intermediate magnitudes that favour the thick disc

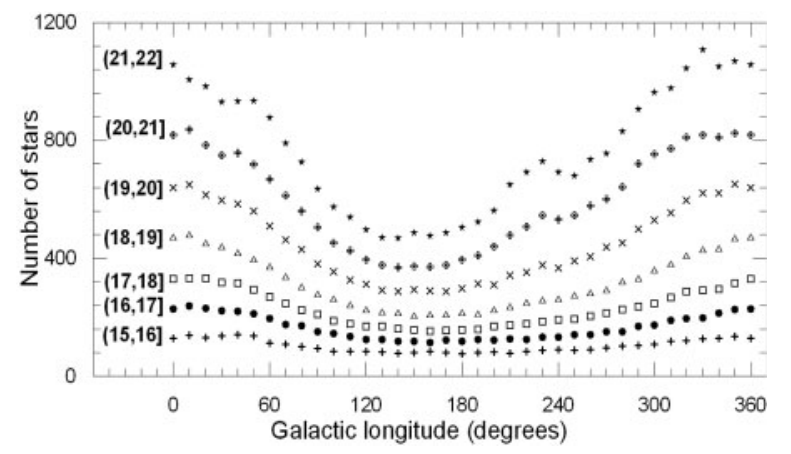

Figure 14 Variation of the number of stars with different $g_{0}$ apparent magnitudes as a function of Galactic longitude.

stars. All the functions have a flat minimum in the interval $120<l<180^{\circ}$, which is more conspicuous at fainter magnitudes. That is, there is a deficiency in the number of stars in that longitude interval. For example, the surface density in this interval is about $50 \%$ less than the one for a field in the direction of the Galactic centre for the apparent magnitude interval $21<g_{0}<22$. The lower surface density for the fields in the anti-centre direction relative to the fields in the Galactic-centre direction is an effect of the disc scalelength. However, this effect should be most efficient in the field with longitude $l=180^{\circ}$, which is not the case in Figure 14. The excess of stars for the fields $l \approx 230^{\circ}$ corresponds to the 'tail of the Sagittarius tidal stream' overdensity region cited by Newberg, Mayeur \& Yanny (2006), that was also noted by Xu et al. (2006).

Following Parker et al. (2003), we compared the number of stars for five pairs of fields which are symmetric relative to the meridian, i.e. the plane perpendicular to the Galactic plane and passing through the centre of the Galaxy and the Sun. The sample pairs are: $\left(30^{\circ}, 330^{\circ}\right),\left(60^{\circ}, 300^{\circ}\right),\left(90^{\circ}, 270^{\circ}\right),\left(120^{\circ}, 240^{\circ}\right)$, and $\left(150^{\circ}, 210^{\circ}\right)$. The comparison is carried out for four colours, i.e. $(g-r)_{0} \leq 0.35,0.35<(g-r)_{0} \leq 0.60$, $0.60<(g-r)_{0} \leq 1.20$, and $1.20<(g-r)_{0}$. As mentioned in the Introduction, blue stars in the range $15<g_{0}<18$ are dominated by thick-disc stars with a turnoff $(g-r)_{0} \approx 0.33$, while halo stars become significant for $g_{0}>18$, with a turn-off $(g-r)_{0} \approx 0.2$. Red stars, $(g-r)_{0} \approx 1.3$ are dominated by thin-disc stars at all apparent magnitudes. Table 3 gives a comparison of the surface densities (number of stars per square degree) for fields in different quadrants as a function of colour and apparent magnitude. We shall treat the ratio of stars given in Table 3 according to their colours. We will use the notation $N_{i} / N_{j}$ for the ratio of stars, for simplicity, where suffixes $i$ and $j$ denote the Galactic longitudes of the fields in question.

The Colour Range $(\boldsymbol{g}-\boldsymbol{r})_{\mathbf{0}} \leq \mathbf{0 . 3 5}$ : Apparently faint stars in this interval are halo stars, whereas the bright ones are (thin or thick) disc stars. The ratio of the number of stars in quadrant $I$ to the number of stars in quadrant IV is about 1 or greater than 1 for bright apparent magnitudes, whereas it decreases when one goes to the fainter magnitudes, and it approaches about 0.5 at $21<g_{0} \leq 22$. The inequality of number of stars for the halo population is due to the 
Table 3. Comparison of the surface densities (number of stars per square degree) for fields in different quadrants $^{\mathrm{a}}$

\begin{tabular}{lcccccc}
\hline$(g-r)_{0}$ & $g_{1}-g_{2}$ & $N_{30} / N_{330}$ & $N_{60} / N_{300}$ & $N_{90} / N_{270}$ & $N_{120} / N_{240}$ & $N_{150} / N_{210}$ \\
\hline$(g-r)_{0} \leq 0.35$ & $15<g_{0} \leq 16$ & 1.55 & 0.88 & 0.91 & 0.83 & 1.18 \\
$(g-r)_{0} \leq 0.35$ & $16<g_{0} \leq 17$ & 0.88 & 1.01 & 0.83 & 0.95 & 1.00 \\
$(g-r)_{0} \leq 0.35$ & $17<g_{0} \leq 18$ & 0.90 & 0.84 & 0.88 & 0.82 & 0.91 \\
$(g-r)_{0} \leq 0.35$ & $18<g_{0} \leq 19$ & 0.87 & 0.88 & 0.80 & 0.75 & 0.85 \\
$(g-r)_{0} \leq 0.35$ & $19<g_{0} \leq 20$ & 0.80 & 0.72 & 0.67 & 0.74 & 0.75 \\
$(g-r)_{0} \leq 0.35$ & $20<g_{0} \leq 21$ & 0.69 & 0.58 & 0.58 & 0.53 & 0.57 \\
$(g-r)_{0} \leq 0.35$ & $21<g_{0} \leq 22$ & 0.47 & 0.57 & 0.56 & 0.38 & 0.45 \\
$0.35<(g-r)_{0} \leq 0.60$ & $15<g_{0} \leq 16$ & 1.16 & 1.08 & 0.99 & 0.95 & 1.02 \\
$0.35<(g-r)_{0} \leq 0.60$ & $16<g_{0} \leq 17$ & 1.14 & 1.15 & 1.04 & 0.96 & 0.92 \\
$0.35<(g-r)_{0} \leq 0.60$ & $17<g_{0} \leq 18$ & 1.11 & 1.16 & 0.94 & 0.84 & 0.92 \\
$0.35<(g-r)_{0} \leq 0.60$ & $18<g_{0} \leq 19$ & 1.01 & 0.94 & 0.83 & 0.78 & 0.78 \\
$0.35<(g-r)_{0} \leq 0.60$ & $19<g_{0} \leq 20$ & 0.83 & 0.82 & 0.74 & 0.71 & 0.75 \\
$0.35<(g-r)_{0} \leq 0.60$ & $20<g_{0} \leq 21$ & 0.75 & 0.71 & 0.69 & 0.60 & 0.74 \\
$0.35<(g-r)_{0} \leq 0.60$ & $21<g_{0} \leq 22$ & 0.61 & 0.67 & 0.67 & 0.57 & 0.67 \\
$0.60<(g-r)_{0} \leq 1.20$ & $15<g_{0} \leq 16$ & 1.04 & 1.06 & 1.02 & 0.97 & 0.93 \\
$0.60<(g-r)_{0} \leq 1.20$ & $16<g_{0} \leq 17$ & 1.26 & 1.16 & 0.91 & 0.88 & 0.95 \\
$0.60<(g-r)_{0} \leq 1.20$ & $17<g_{0} \leq 18$ & 1.15 & 1.18 & 0.98 & 0.95 & 0.91 \\
$0.60<(g-r)_{0} \leq 1.20$ & $18<g_{0} \leq 19$ & 1.06 & 1.11 & 1.03 & 0.88 & 0.89 \\
$0.60<(g-r)_{0} \leq 1.20$ & $19<g_{0} \leq 20$ & 1.03 & 1.07 & 0.97 & 0.88 & 0.90 \\
$0.60<(g-r)_{0} \leq 1.20$ & $20<g_{0} \leq 21$ & 0.98 & 1.02 & 0.92 & 0.80 & 0.89 \\
$0.60<(g-r)_{0} \leq 1.20$ & $21<g_{0} \leq 22$ & 0.93 & 0.88 & 0.83 & 0.79 & 0.91 \\
$(g-r)_{0}>1.20$ & $15<g_{0} \leq 16$ & 0.90 & 1.17 & 0.83 & 0.76 & 1.05 \\
$(g-r)_{0}>1.20$ & $16<g_{0} \leq 17$ & 1.14 & 1.12 & 0.98 & 1.07 & 0.95 \\
$(g-r)_{0}>1.20$ & $17<g_{0} \leq 18$ & 1.17 & 1.22 & 1.18 & 0.90 & 0.90 \\
$(g-r)_{0}>1.20$ & $18<g_{0} \leq 19$ & 1.16 & 1.20 & 1.13 & 1.01 & 0.92 \\
$(g-r)_{0}>1.20$ & $19<g_{0} \leq 20$ & 1.19 & 1.23 & 1.06 & 0.98 & 0.94 \\
$(g-r)_{0}>1.20$ & $20<g_{0} \leq 21$ & 1.20 & 1.26 & 1.15 & 0.99 & 0.89 \\
$(g-r)_{0}>1.20$ & $21<g_{0} \leq 22$ & 1.14 & 1.28 & 1.10 & 0.98 & 0.86 \\
\hline
\end{tabular}

${ }^{a}$ The suffix denotes the longitude of the field in question.

triaxiality of this component of the Galaxy. The same case holds for the halo stars in quadrant II and quadrant III. However, for bright stars, the interpretation of the ratio of stars seems difficult (but see the following sections).

The Colour Range $0.35<(g-r)_{0} \leq \mathbf{0 . 6 0}$ : This is a perfect colour range for the interpretation of the ratios of the number of stars in the corresponding fields, since relatively bright stars belong to the thick disc population whereas the faint ones are halo population objects. The ratios $N_{30} / N_{330}$ and $N_{60} / N_{300}$ are greater than 1 for three apparently bright intervals, i.e. $g_{0}:(15,16],(16,17]$ and $(17,18]$ whereas it is less than 1 for $g_{0}:(19,20],(20$, $21]$ and $(21,22]$. An excess in number of stars in quadrant I relative to quadrant IV can be explained by the existence of a bar with its near end at the Galactic longitude $l \approx 27^{\circ}$ (Parker et al. 2003). According to this argument one expects a deficiency of stars in quadrant II relative to quadrant III. Actually, this is the case in our work, i.e. $N_{120} / N_{240}$ and $N_{150} / N_{210}$ are less than 1 for (thick) disc stars. Here also, the inequality of number of stars with apparently faint magnitudes is due to the triaxiality of the halo. The apparent magnitude interval $18<g_{0} \leq 19$ is a transition region between disc and halo populations.

The Colour Range $0.60<(g-r)_{0} \leq 1.20$ : This is also a perfect colour range for discussing the number of stars in the fields in different quadrants. The ratios $N_{30} / N_{330}$ and $N_{60} / N_{300}$ are greater than 1 for five apparent magnitude intervals i.e. $g_{0}:(15,16],(16,17],(17,18],(18,19]$ and $(19,20]$, indicating a fainter limiting apparent magnitude for the thick-disc stars in this colour range. As expected, the ratios $N_{120} / N_{240}$ and $N_{150} / N_{210}$ are less than 1 for the same apparent magnitude intervals. The combination of these results confirm our argument stated in the previous section, i.e. the inequality of the number of stars brighter than $g_{0}=20$ in quadrant I and quadrant IV originates from the effect of the disc bar. For stars with $g_{0}>20$, all the ratios of the number of stars mentioned above are less than 1 . These stars belong to the population of a triaxial halo, as cited above. We should note that the ratio of the number of faint stars (halo stars) with this colour range is larger than the corresponding ones in the previous colours.

The Colour Range $(g-r)_{0}>$ 1.20: As cited in the previous sections, the red stars belong to the thin disc population. Since the number of stars is rather small, we omit the brightest apparent magnitude interval, $(15,16]$. For the other six intervals, the ratios $N_{30} / N_{330}$ and $N_{60} / N_{300}$ are greater than 1 , as in the previous sections. On the other hand, the ratios $N_{120} / N_{240}$ and $N_{150} / N_{210}$ are less than 1 or equal to 1 . That is, although the asymmetrical structure of the disc can also be explained by the data in this section, 
there is a slight difference between the effects of the bar on the stars in quadrant I and quadrant III.

\subsubsection{Relation Between the Variation of the Galactic Model Parameters and the Asymmetric Structure of the Galaxy}

The global distributions of the Galactic model parameters in Figures 11-13 show minimum and maximum values at different Galactic longitudes, whereas the investigation of these distributions with a short scale reveal interesting sub-structures, i.e. one can separate the plots in a figure into several sub-sets which form segments with different slopes. It is difficult to interpret the dependence of the Galactic model parameters by means of such a distribution. However, this can be done by combining the observed distributions in Figures 11-13 and the results in Section 4.2.1. The difference between the corresponding Galactic model parameters of the halo for different Galactic longitudes confirms the argument that the halo has a triaxial structure. However, for the discs the reason is different. Different surface densities between the fields in quadrants I and IV and in quadrants III and II confirm the effect of a disc bar. The bar induces a gravitational 'wake', traps and piles up stars behind it (Hernquist \& Weinberg 1992; Debattista \& Sellwood 1998). Thus, in response to a bar, one expects an excess of stars in quadrant I (quadrant III) over quadrant IV (quadrant II) which is the case in our work. Also, the disc flarings produce a change in the scaleheight of the thin and thick discs as the galactocentric distance varies. This effect is well explained in López-Corredoira et al. (2002, 2004), and Cabrera-Lavers et al. (2007), who showed that the thick disc presents a flaring in the opposite sense as for the thin disc, that is a decrease in $h_{\mathrm{z}}$ as $R$ increases for the thick disc, whereas there is an increase in $h_{\mathrm{z}}$ with $R$ for the thin disc (López-Corredoira et al. 2002; Momany et al. 2006).

Figure 15 shows the trend in both the thin and thick disc scaleheights with the galactocentric distance. In the upper panel, the solid line represents the change in the scaleheight of the thin disc obtained by López-Corredoira et al. (2002), (Cabrera-Lavers et al. 2007, Equation 4), with $h_{\mathrm{z}}\left(R_{\odot}\right)=185 \mathrm{pc}$. It is clear that the results obtained are compatible with this flaring, but the scatter is too high to be conclusive in this statement. Also, the range of galactocentric distances covered by the data is too small to infer a proper fit from the data. In the lower panel, the solid line shows the relationship found in Cabrera-Lavers et al. (2007, their eq. 5 ) by using $2 M A S S$ data at latitudes $\langle b\rangle=65^{\circ}$, but now considering $h_{\mathrm{z}}\left(R_{\odot}\right)=700 \mathrm{pc}^{3}$. Data for $R>8 \mathrm{kpc}$ are compatible with the trend previously obtained, although there is a decrease in the values for the innermost data with respect to the predictions of this

\footnotetext{
${ }^{3}$ Both scaleheights in the solar neighbourhood are dependent on the populations under consideration, so a slight decrease in those values with respect to that obtained for the giant population, dominant in the $2 M A S S$ data, is not unexpected.
}
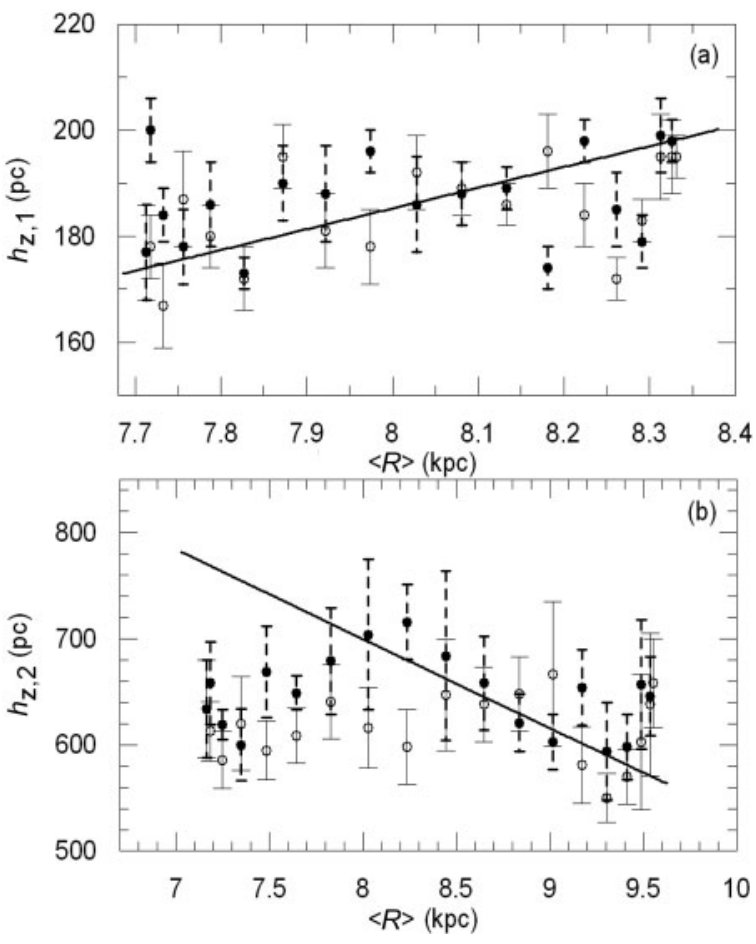

Figure 15 Variation of the scaleheight of the thin (a) and thick (b) discs with $\langle R\rangle$. The symbols (o) and (•) correspond to the data for the fields with Galactic longitudes $0<l \leq 180^{\circ}$ and $180<l \leq 360^{\circ}$, respectively. The error bars for the fields with $180<l \leq 360^{\circ}$ are marked with a different symbol (dashed segment) in order to separate them from the error bars of the fields with $0<l \leq 180^{\circ}$.

law that would need a further explanation. Therefore, it seems that the observed trend in the scaleheight of the thick disc via the $2 M A S S$ data is also reproducible with the $S D S S$ data. Hence the disc flarings have an important contribution to the observed changes in the parameters with galactic longitude, as different longitudes imply different ranges of galactocentric distances.

The two points for each $\langle R\rangle$ bin shown in Figure 15 correspond to the different scaleheights of the two symmetric fields relative to the meridian. This confirms the asymmetric structure of the thin (upper panel) and thick (lower panel) discs. The different behaviours of the radial variation of the scaleheights of thin and thick discs allow specific predictions about the radial dependence of the vertical velocity dispersion of the discs, since at a given $\langle R\rangle$ they are in the same gravitational field. The different behaviours are real, and, as cited at the end of the first paragraph of this section, they are due to the opposite sense of the flaring of two discs, i.e. $h_{\mathrm{z}}$ decreases as $R$ increases for the thick disc, whereas there is an increase in $h_{\mathrm{z}}$ with $R$ for the thin disc. The variations of the scaleheights of thin and thick discs with Galactic longitude are also different (Figure 11 and the upper panel of Figure 12). Additionally, one can see more easily that the scaleheights of thin or thick discs for two fields that are symmetric relative to the meridian are different which, again, confirms the asymmetrical structure of the two discs. 


\section{Conclusion}

The dependence of the Galactic model parameters on Galactic longitude can be explained as a result of the combined effects of the triaxial structure of the halo, the gravitational effect of the disc bar and the thin- and thickdisc flarings, which are more dominant in some Galactic coordinates than with respect to others.

\section{A Two-Colour Diagram}

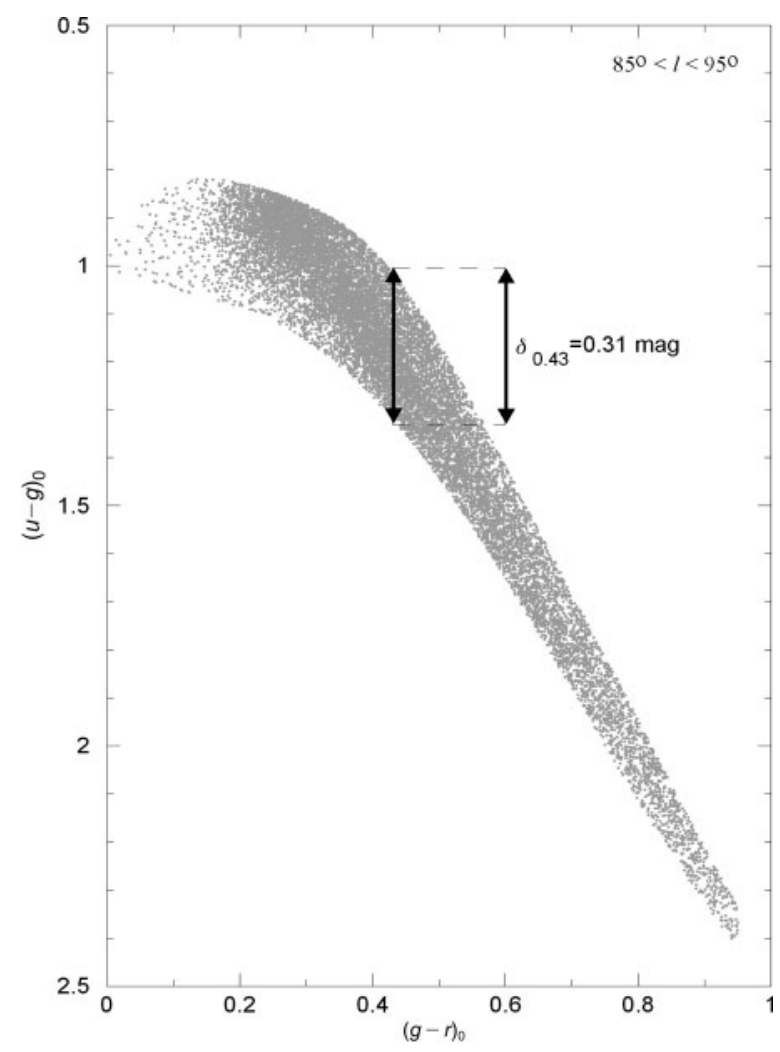

Figure $16(u-g)_{0},(g-r)_{0}$ two-colour diagram for stars in the field with longitude $l=90^{\circ}$. The difference in $U V$-excess between the upper and lower envelopes of the diagram is small at the red end of the diagram which causes an uncertainty in the metallicity estimation. The maximum $U V$-excess, $\Delta(u-g)=0.31 \mathrm{mag}$, corresponds to the colour $(g-r)_{0}=0.43 \mathrm{mag}$ which is equivalent to $(B-V)_{0}=0.60 \mathrm{mag}$ in the $U B V$ photometry.

\section{Acknowledgments}

Thanks are given to an anonymous referee, who made very important suggestions that have improved the overall quality of the work presented in this paper. This work was supported by the Research Fund of the University of Istanbul: Project number: BYP 941-02032006. We thank Hikmet Çakmak and Tuğkent Akkurum for preparing some computer codes for this study. S. Karaali thanks to T. C. Beykent University for financial support.

The SDSS is managed by the Astrophysical Research Consortium (ARC) for the Participating Institutions. The Participating Institutions are The University of Chicago, Fermilab, the Institute for Advanced Study, the Japan Participation Group, The Johns Hopkins University, the
Korean Scientist Group, Los Alamos National Laboratory, the Max-Planck-Institute for Astronomy (MPIA), the Max-Planck-Institute for Astrophysics (MPA), New Mexico State University, University of Pittsburgh, University of Portsmouth, Princeton University, the United States Naval Observatory, and the University of Washington. Funding for the creation and distribution of the SDSS Archive has been provided by the Alfred P. Sloan Foundation, the Participating Institutions, the National Aeronautics and Space Administration, the National Science Foundation, the U.S. Department of Energy, the Japanese Monbukagakusho, and the Max Planck Society. The SDSS Web site is http: //www. sdss . org/.

\section{References}

Adelman-McCarthy, J. K. et al., 2007, ApJS, 172, 634

Ak, S., Bilir, S., Karaali, S., Buser, R. \& Cabrera-Lavers, A., 2007a, NewA, 12, 605

Ak, S., Bilir, S., Karaali, S. \& Buser, R., 2007b, AN, 328, 169

Alves, D. R., 2000, ApJ, 539, 732

Bahcall, J. N., 1986, ARA\&A, 24, 577

Becker, W., 1965, ZA, 62, 54

Bertin, A. \& Arnouts, S., 1996, A\&AS, 117, 393

Blanton, M. R. et al., 2005, AJ, 129, 2562

Bilir, S., Karaali, S. \& Tunçel, S., 2005, AN, 326, 321

Bilir, S., Karaali, S., Ak, S., Yaz, E. \& Hamzaoğlu, E., 2006a, NewA, 12,234

Bilir, S., Karaali, S. \& Gilmore, G., 2006b, MNRAS, 366, 1295

Bilir, S., Karaali, S., Güver, T., Karataş, Y. \& Ak, S., 2006c, AN, 327, 72

Burton, W. B., 1988, Galactic and Extragalactic Radio Astronomy, 2nd version, Eds. Verschuur, G. L. \& Kellerman, K. I. (Berlin: Springer-Verlag)

Buser, R. \& Fenkart, R., 1990, A\&A, 239, 243

Buser, R., Rong, J. \& Karaali, S., 1998, A\&A, 331, 934

Buser, R., Rong, J. \& Karaali, S., 1999, A\&A, 348, 98

Cabrera-Lavers, A., Bilir, S., Ak, S., Yaz, E. \& López-Corredoira, M., 2007, A\&A, 464, 565

Carney, B. W., 1979, ApJ, 233, 211

Chen, B. et al., 2001, ApJ, 553, 184

de Vaucouleurs, G., 1948, AnAp, 11, 247

Debattista, V. P. \& Sellwood, J. A., 1998, ApJ, 493, L5

Drimmel, R. \& Spergel, D. N., 2001, ApJ, 556, 181

Du, C. et al., 2003, A\&A, 407, 541

Du, C., Ma, J., Wu, Z. \& Zhou, X., 2006, MNRAS, 372, 1304

Fan, X., 1999, AJ, 117, 2528

Fenkart, R., 1989a, A\&AS, 78, 217

Fenkart, R., 1989b, A\&AS, 79, 51

Fenkart, R., 1989c, A\&AS, 80, 89

Fenkart, R., 1989d, A\&AS, 81, 187

Fukugita, M., Ichikawa, T., Gunn, J. E., Doi, M., Shimasaku, K. \& Schneider, D. P., 1996, AJ, 111, 1748

Gilmore, G. \& Reid, N., 1983, MNRAS, 202, 1025

Gilmore, G., Wyse, R. F. G. \& Kuijken, K., 1989, ARA\&A, 27, 555

Gilmore, G., Wyse, R. F. G. \& Jones, J. B., 1995, AJ, 109, 1095

Helmi, A. et al., 2003, ApJ, 586, 195

Hernquist, L. \& Weinberg, M. D., 1992, ApJ, 400, 80

Ivezic, Z., Lupton, R. H., Schlegel, D., Boroski, B. et al., 2004, AN, 325,583

Jahreiss, H. \& Wielen, R., 1997, in HIPPARCOS - Venice '97 (ESA SP-402), Eds. Battrick, B., Perryman, M. A. C. \& Bernacca, P. L., 675

Jurić, M. et al., 2008, ApJ, 673, 864

Karaali, S., Bilir, S. \& Hamzaoğlu, E., 2004, MNRAS, 355, 307

Karaali, S., Bilir, S. \& Tunçel, S., 2005, PASA, 22, 24 
Karaali, S., Bilir, S., Yaz, E., Hamzaoğlu, E. \& Buser, R., 2007, PASA, 24, 208

Kent, S. M., Dame, T. M. \& Fazio, G., 1991, ApJ, 378, 131

Levine, E. S., Blitz, L. \& Heiles, C., 2006, ApJ, 643, 881

López-Corredoira, M., Cabrera-Lavers, A., Garzón, F. \& Hammersley, P. L., 2002, A\&A, 394, 883

López-Corredoira, M., Cabrera-Lavers, A., Gerhard, O. \& Garzón, F., 2004, A\&A, 421, 953

Lupton, R. H., Gunn, J. E., Ivezic, Z., Knapp, G. R., Kent, S. \& Yasuda, N., 2001, in ASPC: Astronomical Data Analysis Software and Systems X, Eds. Harden, F. R., Jr., Primini, F. A. \& Payne, H. E., 238, 269

Malmquist, G., 1920, MeLu2, 22

Majewski, S. R., 1993, ARA\&A, 31, 575

Marshall, D. J., Robin, A. C., Reylé, C., Schultheis, M. \& Picaud, S., 2006, A\&A, 453, 635

Momany, Y., Zaggia, S. R., Gilmore, G., Piotto, G., Carraro, G., Bedin, L. R. \& De Angeli, F., 2006, A\&A, 451, 515

Nakanishi, H. \& Sofue, Y., 2003, PASJ, 55, 191

Narayan, C. A. \& Jog, C. J., 2002, A\&A, 394, 89

Newberg, H. J. \& Yanny, B., 2005, in ASPC: Astrometry in the Age of the next Generation of Large Telescopes, Eds. Seidelmann, P. K. \& Monet, A. K. B., 338, 210

Newberg, H. J. \& Yanny, B., 2006, Journal of Physics: Conference Series, 47, 195
Newberg, H. J., Mayeur, P. A. \& Yanny, B., 2006, MmSAI, 77, 1049 Ojha, D. K., Bienaymé, O., Mohan, V. \& Robin, A. C., 1999, A\&A, 351,945

Parker, J. E., Humphreys, R. M. \& Larsen, J. A., 2003, AJ, 126, 1346

Phleps, S., Meisenheimer, K., Fuchs, B. \& Wolf, C., 2000, A\&A, 356, 108

Phleps, S., Drepper, S., Meisenheimer, K. \& Fuchs, B., 2005, A\&A, 443, 929

Pier, J. R., Munn, J. A., Hindsley, R. B., Hennessy, G. S., Kent, S. M., Lupton, R. H. \& Ivezic, Z., 2003, AJ, 125, 1559

Reid, M. J., 1993, ARA\&A, 31, 345

Robin, A. C., Haywood, M., Crézé, M., Ojha, D. K. \& Bienaymé, O., 1996, A\&A, 305, 125

Robin, A. C., Reylé, C. \& Crézé, M., 2000, A\&A, 359, 103

Schlegel, D. J., Finkbeiner, D. P. \& Davis, M., 1998, ApJ, 500, 525

Siegel, M. H., Majewski, S. R., Reid, I. N. \& Thompson, I. B., 2002, ApJ, 578, 151

Voskes, T. \& Burton, W. B., 2006, MSc Thesis, University of Leiden (astro-ph/0601653)

Wyse, R. F. G. \& Gilmore, G., 2005, astro-ph/0510025

Xu, Y., Deng, L. C. \& Hu, J. Y., 2006, MNRAS, 368, 1811

Young, P. J., 1976, AJ, 81, 807

York, D. G. et al., 2000, AJ, 120, 1579 